\title{
OPINION
}

\section{Galectins in prostate and bladder cancer: tumorigenic roles and clinical opportunities}

Neus Martínez-Bosch, Alejo Rodriguez-Vida, Núria Juanpere, Josep Lloreta, Ana Rovira, Joan

Albanell, Joaquim Bellmunt and Pilar Navarro

\begin{abstract}
Advanced prostate and bladder cancer are two outstanding unmet medical needs for urological oncologists. The high prevalence of these tumours, lack of effective biomarkers, and limited effective treatment options highlight the importance of basic research in these diseases. Galectins are a family of $\beta$-galactoside-binding proteins that are frequently altered (upregulated or downregulated) in a wide range of tumours and have roles in different stages of tumour development and progression, including immune evasion. In particular, altered expression levels of different members of the galectin family have been reported in prostate and bladder cancers, which, together with the aberrant glycosylation patterns found in tumour cells and the constituent cell types of the tumour microenvironment, can result in malignant transformation and tumour progression. Understanding the roles of galectin family proteins in the development and progression of prostate and bladder cancer could yield key insights to inform the clinical management of these diseases.
\end{abstract}

\section{[H1] Introduction}

Genitourinary cancers pose a major clinical challenge. Indeed, the urological system is among the most common sites of new cancer diagnoses in developed countries, and prostate and bladder cancer are two of the most frequently diagnosed genitourinary neoplasms ${ }^{1}$.

Important advances in preclinical research ${ }^{2}$ and clinical management ${ }^{3-6}$ have been made over the past five years in the field of prostate cancer, including large-scale DNA sequencing analyses of prostate cancer tumours ${ }^{7}$, the use of patient derived xenograft (PDX) models and organoids ${ }^{2}$, the development of transgenic preclinical models ${ }^{8}$, and the advent of liquid biopsies ${ }^{9}$. Advances in the clinical setting have included the incorporation of docetaxel and abiraterone in the metastatic castration-sensitive prostate cancer (CSPC) setting, the approvals of enzalutamide and apalutamide in the non-metastatic castration-resistant prostate cancer (CRPC) setting, and the advent of next-generation molecular PET imaging such as ${ }^{68} \mathrm{Ga}$-prostate-specific membrane 
antigen (PSMA) PET-CT ${ }^{6}$. Nevertheless, prostate cancer is still the most commonly diagnosed cancer in men and the second most common cause of male cancer-related mortality in developed countries $^{1}$. Major clinical challenges in prostate cancer include accurate risk stratification and determining which (if any) treatment options should be offered at each disease stage. Moreover, current biomarkers used for diagnosis, risk stratification, prognostication, and prediction of recurrence (for example, serum PSA levels and needle biopsy-determined Gleason score) present several limitations, such as false-negative and/or false-positive results and inexact predictions, which lead to overdiagnosis and unnecessary treatment of patients with low-risk prostate cancer ${ }^{10,11}$. Thus, new molecular markers and improved therapeutic approaches are urgently needed to improve discrimination between indolent and aggressive localized tumours and manage patients with advanced-stage disease. Current treatments for localized prostate cancer include radical prostatectomy or radiotherapy with or without androgen deprivation therapy $(A D T)^{6}$. However, disease recurrence and emergence of CRPC is frequent after therapy, for which treatment options are still insufficient and survival rates are still low in the advanced setting ${ }^{12}$. In addition, although immune checkpoint inhibitor (ICI) immunotherapy has received approval from the FDA and European Medicines Agency (EMA) for treatment of other genitourinary neoplasms, such as renal cell carcinoma (RCC) and bladder cancer ${ }^{13-18}$, such treatments are not available for patients with prostate cancer patients as they have shown limited success in clinical trials with unselected cohorts $^{19}$.

Bladder cancer is the fourth most commonly diagnosed cancer and the eighth most common cause of cancer-related death for men in developed countries ${ }^{1}$, making it a major public health concern. Most bladder tumours (78-85\%) are non-muscle-invasive bladder cancers (NMIBCS) that are confined to the mucosa or submucosa and are associated with good survival outcomes ${ }^{20}$; however, as NMIBCs have a high risk of recurrence, they require life-long treatment. Treatment options for NMIBC include transurethral resection of the bladder tumour (TURBT) followed by intravesical instillations of Bacillus Calmette-Guerin (BCG) immunotherapy or mitomycin, and radical cystectomy for high-grade recurring NMIBCs ${ }^{21}$. By contrast, muscle-invasive bladder cancers (MIBCs) present a more urgent clinical need as the 5-year survival remains $\sim 50 \%{ }^{22}$. In this setting, radical cystectomy preceded by neoadjuvant chemotherapy is considered the standard therapy in patients deemed fit, although researchers and clinicians are now also considering bladder-sparing approaches ${ }^{23}$. The advent of immunotherapy with ICls has been a major breakthrough in bladder cancer and has become a new standard of care for patients with 
metastatic disease in the second line setting ${ }^{15}$ and a valid option in the first-line setting for patients with programmed cell death 1 ligand 1 (PD-L1)-positive metastatic disease who are ineligible for cisplatin ${ }^{24}$. In addition, ICls are currently being tested in clinical trials in localized disease (NMIBC and $\mathrm{MIBC}$ ) and in combination with chemotherapy in the metastatic first-line setting ${ }^{24}$.

The unpredictability of long-term outcomes among patients with bladder cancer or prostate cancer highlights the need for an increased molecular understanding of these diseases to improve clinical management in terms of diagnosis, prognostication, and therapy ${ }^{25}$. In line with the goal of translating basic research into promising new therapies, emerging data have established a role for galectins in prostate and bladder cancer development and progression, providing a number of clinical opportunities for development of new therapies for both diseases. In addition, several groups have addressed the potential of galectins as biomarkers to improve diagnosis and prognostication in prostate and bladder cancer ${ }^{26-35}$. Galectins are a family of proteins that structurally share a carbohydrate recognition domain (CRD) and have a high affinity for glycan structures that contain lactosamine residues ${ }^{36}$. These proteins are expressed by different cell types and have been reported to mediate functions in development, tissue regeneration and cancer ${ }^{36}$. However, the wide range of biological functions of this family of proteins, as well as their overlapping roles in mediating certain biological functions ${ }^{37-39}$, pose an important obstacle in understanding these proteins and exploiting this knowledge to improve the management of patients with prostate cancer or bladder cancer.

In this Opinion, we address how aberrant glycosylation and abnormal galectin expression cooperate to drive tumour initiation and progression, with a particular focus on the emerging roles of galectins in prostate and bladder cancer. We highlight the roles of the best-studied galectin family members, galectin-1 (Gal-1) and galectin-3 (Gal-3), as well as the putative roles of other family members that have been investigated superficially or not at all. Finally, we speculate regarding how this knowledge could be potentially translated to the clinical setting.

\section{[H1] Glycosylation and galectins in cancer}

Altered glycosylation is a key feature of cancer, including prostate and bladder cancer ${ }^{40,41}$, and can be exploited for cancer therapy and diagnosis ${ }^{42,43}$. Galectins have emerged as an important family of proteins with roles in tumour development and progression, and can detect and translate tumour glycosylation to influence cellular interactions, cell signalling, and pathological outcomes ${ }^{44}$. 


\section{[H2] Aberrant glycosylation in cancer}

Malignant transformation is often accompanied by alterations in cellular glycosylation, an enzymatic process involving the attachment of carbohydrate moieties (glycans) to proteins that is involved in tumour development and progression and that, therefore, could inform cancer diagnosis and therapy ${ }^{42}$. Importantly, these changes in protein glycosylation affect all components of a tumour, including epithelial tumour cells, cancer-associated fibroblasts, immune cells, endothelial cells and extracellular matrix $(\mathrm{ECM})^{42,43,45,46}$. The two basic processes that guide aberrant glycosylation in cancer are the incomplete synthesis of glycans and the neosynthesis of cancer-associated cell surface glycans ${ }^{47}$. These changes can occur as a result of downregulation, overexpression, or even mislocalization of glycosyltransferases in tumour cells, but also depend on the abundance of sugar nucleotide donors or the tertiary structure of the glycoprotein backbone ${ }^{43}$. The most common glycan-specific aberrant structures that occur during tumorigenesis include altered branching and fucosylation of $\mathrm{N}$-glycans, truncation of $\mathrm{O}$-glycans, altered expression and glycosylation of mucins, and altered sialic acid expression ${ }^{43,48}$. Glycans modulate protein structure and 3D conformation and, therefore, these alterations, which are typically found in tumour cells, translate into a range of different cell-cell interactions and signaling mechanisms that can regulate biological processes during cancer progression and development, such as cell-cell adhesion, cellmatrix interactions, tumour immune surveillance and cancer metabolism ${ }^{43}$. For instance aberrant glycosylation and increased sialylation of E-cadherin results in cell detachment, owing to malfunction of adherens junctions ${ }^{49}$ or electrostatic repulsion ${ }^{50}$, respectively. Moreover, altered CD44 glycosylation at the tumour cell surface controls its interaction with hyaluronic acid ${ }^{51}$, and changes in integrin $\mathrm{N}$-glycans regulate their functions $\mathrm{s}^{52}$. Antibodies raised against altered patterns of $O$-Glycosylation on the cancer cell membrane, which trigger antibody-dependent cellular cytotoxicity, are candidates for cancer-specific immunotherapies ${ }^{53}$.

Aberrant glycan profiles have been described in prostate and bladder cancers ${ }^{40,41,54}$, and interest has been growing in the identification of biomarkers linked to proteins with altered glycosylation ${ }^{55,56}$. Interestingly, monitoring changes in serum PSA glycosylation has been suggested as a method to improve the diagnosis of prostate cancer, as this approach could increase sensitivity of the current standard PSA detection, with the advantage of being able to discriminate between benign and malignant states of the disease ${ }^{55,57-59}$. Differential glycosylation of urine or serum markers has also been considered for bladder cancer diagnostics. Immuncytostaining with 
antibodies against Lewis $\mathrm{X}$ - a blood group antigen that is absent in normal urothelial cells but expressed in bladder cancer cells - in cells from urine samples has proven to be particularly interesting for identifying low-grade bladder carcinomas and predicting tumour progression and recurrence ${ }^{60}$. In addition, a particular pattern of aberrant glycosylation in serum immunoglobulins successfully detected patients with bladder cancer from controls and from patients with prostate cancer $^{56}$. Moreover, detection of urine hyaluronic acid, a glycosaminoglycan that is overexpressed in bladder cancer, in combination with measurement of hyaluronidase levels (the HA-HAase test), achieved a $91.2 \%$ sensitivity and an $84.4 \%$ specificity for bladder cancer detection ${ }^{61}$. However, further investigation will be required to fully understand the role of glycosylation in prostate and bladder cancers and how these alterations can be exploited for diagnosis, treatment, and followup monitoring.

\section{[H2] The galectin family}

Galectins are efficient 'translators' that decode the information contained within the versatile and dynamic tumour glycome into cellular functions ${ }^{62}$. Galectins comprise a family of 15 proteins in mammals (11 of which exist in humans: Gal-1, Gal-2, Gal-3, Gal-4, Gal-7, Gal-8, Gal-9, Gal-10, Gal12, Gal-13, Gal-14) that share a highly conserved CRD and typically bind structures with lactosamine residues present in glycoconjugates ${ }^{36}$. Galectins are classified into three subfamilies on the basis of the number and organization of their CRDs, including: prototype galectins (Gal-1, Gal-2, Gal-5, Gal-7, Gal-10, Gal-11, Gal-13, Gal-14, and Gal-15), which contain a single CRD and can function as monomers or dimerize; tandem-repeat galectins (Gal-4, Gal-6, Gal-8, Gal-9, and Gal12), which have two CRDs that are held together by a short linker peptide; and a chimeric-type galectin (Gal-3) that has an extended N-terminal tail that enables oligomerization ${ }^{63}$ (FIG. 1).

Different members of the galectin family have distinct ligand selectivity and interaction strengths and are regulated at multiple levels, such as by their state of oligomerization, the environmental redox conditions, and their subcellular or extracellular localization ${ }^{44,64-66}$. Galectin oligomerization enables CRD clustering and, subsequently, crosslinking of glycoproteins or glycolipids on the cell membrane, resulting in lattice formation, which activates cell signalling pathways that lead to several cellular functions, such as cell activation, differentiation and survival, and also controls receptor endocytosis and turnover, immune cell activation, or host-pathogen interactions ${ }^{67,68}$. Furthermore, the redox status of the environment modulates the affinity of galectin for their ligands, with most of them becoming active in a reducing context ${ }^{69}$. For instance, 
Gal-1 requires a dimeric conformation (determined by high Gal-1 concentrations and a reducing environment) for its extracellular functions and crosslinking activities ${ }^{70}$, but requires as a monomeric conformation for its intracellular roles, which are mostly mediated through proteinprotein interactions (for instance, Gal-1 and Gal-3 interactions with RAS ${ }^{71,72}$ ) and sometimes through protein-carbohydrate recognition ${ }^{39,70,73,74}$ (for example, splicing activity is sensitive to saccharide ligand inhibitors $\left.{ }^{39,75}\right)$.

As the 15 galectins can have a multitude of binding partners and can induce distinct biological effects, galectins have key roles in a number of biological processes, such as early embryogenesis and cell differentiation, tissue regeneration, and regulation of immune responses ${ }^{36}$. Moreover, galectins are soluble molecules that lack a signal peptide and are secreted via a non-conventional route ${ }^{76}$, facilitating their roles in an extensive variety of intracellular and also extracellular activities, such as cell-matrix adhesion or cell-cell adhesion ${ }^{77,78}$. Galectin secretion has enabled its detection in human bodily fluids such as plasma, serum, urine and semen $^{79-81}$, which has important implications for pathological diagnosis and follow-up monitoring, particularly for prostate cancer (which arises in a secretory gland) and bladder cancer (which is exposed to urine).

In addition to the complexity of galectin diversity, glycan repertoires can differ depending on the cellular context, which can lead to different or even opposite functional effects in response to a specific galectin in different cell types. For instance, Gal-1 can have either a positive or a negative effect on cell proliferation, depending on the galectin levels, cellular location, conformation state, as well as the cell type ${ }^{74}$; low Gal-1 concentrations induce dimer formation and CRD recognition resulting in a mitogenic effect, whereas high concentrations of monomeric Gal-1 inhibit cell proliferation independently of sugar recognition. Indeed, Gal-1 is mitogenic in normal vascular smooth muscle cells ${ }^{74}$, endothelial cells ${ }^{82,83}$, hepatic stellate cells ${ }^{74}$, and different tumour cells (glioma ${ }^{84}$ and pancreatic cancer ${ }^{85}$ cells), but it inhibits neuroblastoma cell proliferation and induces apoptosis in activated $\mathrm{T}_{\text {cells }}{ }^{74}$. This galectin multifunctionality has been reported not only for cell proliferation but also in the regulation of cell migration, invasion, survival, cell death and immune system homeostasis ${ }^{36,86-88}$.

Importantly, Gal-3 can undergo post-translation modifications such as proteolysis and phosphorylation ${ }^{89}$. Tyrosine and serine phosphorylation of Gal-3 influences its sugar binding ability and modulates binding affinities to its ligands ${ }^{90}$, therefore regulating biological functions such as 
its antiapoptotic function or cell cycle arrest ${ }^{91}$. Moreover, proteolytic cleavage of Gal-3 abrogates multivalence, preserving lectin activity ${ }^{89}$.

\section{[H2] Galectins in cancer development and progression}

Galectin expression is tightly regulated not only under physiological conditions but also in pathological settings, such as inflammation and cancer ${ }^{92-94}$. Galectin activity in cancer was first described in 1975 by Teichberg and colleagues $^{95}$, who reported high levels of a $\beta$-D-galactoside binding protein in a neuroblastoma cell line, and was further linked to tumorigenesis by other investigators during the subsequent decades ${ }^{96,97}$. Galectin overexpression, their roles in driving cancer progression ${ }^{88}$, and their potential as targets for cancer therapy have been studied in detail in different tumour types over the past 10 years ${ }^{81}$. Importantly, galectins have a wide range of roles in cancer including regulation of the processes of cell proliferation, malignant transformation, angiogenesis, migration, invasion, metastasis, and tumour immune evasion $^{44,45,88,94}$ (FIG. 2). The molecular mechanisms responsible for these protumorigenic functions have been partially identified, mainly for Gal-1 or Gal-3, the best studied galectins in cancer to date. These mechanisms include ligand-independent activation of vascular endothelial growth factor receptor 2 (VEGFR2) to induce tumour angiogenesis ${ }^{45}$; interaction with the oncogenic GTPase H-RAS to promote membrane anchorage and cell transformation ${ }^{71,98}$; binding to extracellular proteins (such as laminin and fibronectin) ${ }^{99}$ and integrins ${ }^{100}$ to induce cell-cell and cell-matrix adhesion and migration and/or invasion; regulation of the $\beta$-catenin-WNT ${ }^{101,102}$ or hedgehog signalling pathways ${ }^{103}$; and direct induction of apoptosis in T-effector ( $T_{\text {eff }}$ ) cells by binding to the CD45 and CD7 membrane receptors ${ }^{104,105}$, among other mechanisms ${ }^{88,106}$.

Interestingly, aberrant galectin expression has been reported in $>80 \%$ of human tumour types $^{81}$. The vast majority of studies have focused on Gal-1 and Gal-3 expression, but the interest in different galectins in cancer is growing ${ }^{107}$. Some galectins have shared ligands and, therefore, redundant roles for some galectins in particular settings have been proposed on the basis of observed compensatory actions of galectins in vitro and in vivo. For example, Gal-1 and Gal-3 are redundant splicing factors, Gal-1 and Gal-8 are functionally redundant in promoting plasma cell formation, and Gal-1 and Gal-3 are functionally redundant in placenta implantation ${ }^{37,108,109}$. Nonetheless, the differential and selective patterns of galectin expression in different tumours suggest a high degree of galectin specificity and intricate binding requirements. Importantly, increased Gal-1 levels in tumour tissues (from patients with pancreatic, liver, colon, breast, 
ovarian, lung, prostate, or bladder cancer, among others) or blood samples (from patients with lung, thyroid, glioma, colon, lymphoid, or pancreatic cancer) have been consistently linked with cancer progression and adverse tumour clinicopathological characteristics, and are markers of poor prognosis in many different tumour types ${ }^{81,110}$. Overexpression of Gal-1 is linked to poor patient outcomes in several tumour types (including prostate, gastric, ovarian, pancreatic, and oral squamous cell carcinoma) $)^{81}$, whereas the clinical influence of upregulation or downregulation of other galectins (such as Gal-3 or Gal-9) seems to be tumour-type dependent ${ }^{81,88}$. Finally, considering the important role of several members of the galectin family in tumour progression, the development of galectin-specific pharmacological modulators has received strong interest for therapeutic applications in cancer ${ }^{111}$.

\section{[H1] Galectins in prostate cancer}

The role of galectins in prostate cancer biology has been described ${ }^{112,113}$ (TABLE 1, FIG. 2). These studies have mainly focused on Gal-1 and Gal-3, but the importance of Gal-4, Gal-7, Gal-8, and Gal9 has also been highlighted in this disease setting.

\section{[H2] Gal-1 in prostate cancer}

Gal-1 was first reported to be expressed in prostate cancer cell lines and resident fibroblasts, in basal and luminal cells present in normal prostate tissues, in prostatic intraepithelial neoplasia (PIN), and in primary adenocarcinomas ${ }^{114,115}$. Heterogeneous Gal-1 expression patterns with positive and negative cells detected around the glands were observed in primary adenocarcinomas, whereas more homogenous positivestainings were seen in metastatic sites. Gal1 levels were reported to be increased in prostate cancer cell lines and primary tissues compared with benign hyperplasia, with increasing expression with TNM grade ${ }^{112}$. Studies using larger patient cohorts described Gal-1 overexpression in prostate tumours, particularly in cancerassociated fibroblasts and tumour-associated capillaries, compared with tumour-adjacent normal stroma $^{112,116,117}$.

The proangiogenic role of Gal-1 in prostate cancer-associated vasculature and its link to T cell transendothelial migration has been studied in vitro and in vivo ${ }^{112,116,118}$. Gal-1 expression correlates with blood vessel density ${ }^{112}$ and Gal-1 downregulation in prostate cancer cells reduces tumour angiogenesis in vitro and in vivo ${ }^{112}$. In vitro data showed that tumour cells can upregulate Gal-1 expression in endothelial cells, enhancing adhesion between these two cell types ${ }^{116}$, and that 
Gal-1 inhibits T cell migration across endothelial cells by clustering of CD43 ${ }^{118}$. Androgen-refractory prostate cancer cells can evade Gal-1-driven apoptosis through the overexpression of sialyl-Tn antigen (a truncated O-glycan overexpressed in cancer) produced by ST3 $\beta$-galactoside alpha-2,3sialyltransferase 1 (ST3GAL1) ${ }^{119,120}$, resulting in cancer cell survival and specific $T$ cell apoptosis leading to evasion of immune surveillance. Another study demonstrated that Gal-1 knockdown impaired prostate cancer cell proliferation, anchorage-independent growth, migration, and invasion, and the use of an allosteric Gal-1 inhibitor in vivo potentiated the efficacy of docetaxel chemotherapy and inhibited tumour invasion and metastasis in xenograft models ${ }^{121}$. In addition, Gal-1 in prostate cancer cells has been reported to induce osteoblast proliferation and differentiation, influencing matrix mineralization during bone metastasis ${ }^{122}$. Interestingly, Jaworski et al. ${ }^{123}$ performed a bioinformatics analysis using previously published microarray datasets from Oncomine and identified patients with localized or metastatic prostate cancer whose tumours had moderate heme oxygenase-1 (HO-1; a modulator of angiogenesis and immune function) and low Gal-1 expression as a patient subgroup that could potentially be treated with antiangiogenic and/or immune-targeted therapy. Considering the relevance of Gal-1 and HO-1 in prostate cancer neovascularization and immune response modulation, this subset could be less prone to resistance to these drugs than patients whose tumours have high Gal-1 expression. Moreover, data from 2018 have shown that endogenous Gal-1 in lymphocytes decreased the proliferation and cytotoxic activity of $\mathrm{CD}^{+} \mathrm{T}$ cells, thereby abrogating antitumour immune responses in prostate cancer ${ }^{124}$.

In summary, although the tissue pattern of Gal-1 expression has raised controversy in prostate cancer, Gal-1 has consistently been found to be overexpressed in tumours, regulating important features of prostate cancer progression, such as angiogenesis and tumour immune evasion.

\section{[H2] Gal-3 in prostate cancer}

Analyses of Gal-3 expression at the mRNA and protein levels in localized and metastatic CSPC and/or CRPC tissue samples have consistently found Gal-3 downregulation in tumour cells $^{30,112,114,115,125-131}$, which has been attributed to promoter methylation ${ }^{30,132}$. However, specific Gal-3 cytoplasmic overexpression in tumour cells has also been positively associated with disease progression $^{131}$. A possible explanation for this apparent contradiction might be the dual role of Gal-3 in prostate cancer cells, depending on its subcellular localization. Indeed, cytoplasmic Gal-3 
promotes tumour development, whereas nuclear Gal-3 expression might have anti-tumour activities $^{133}$.

In vitro studies have revealed that Gal-3 can inhibit apoptosis in prostate cancer cells ${ }^{29,134-}$ 136 and induce $\mathrm{T}$ cell apoptosis ${ }^{137}$, tumour cell adhesion to endothelial cells ${ }^{138,139}$, proliferation, migration, and invasion of prostate tumour cells ${ }^{127,136,140}$. These findings were corroborated in vivo using tumour xenograft mouse models, in which Gal-3 inhibition with pharmacological or RNA interference (RNAi) strategies impaired tumour growth ${ }^{127,136}$, angiogenesis ${ }^{137}$, and metastasis ${ }^{29,137,141}$. Importantly, Gal-3 secreted from prostate cancer cells promoted prostate cancer bone metastasis ${ }^{142,143}$ by remodelling the osteolytic bone tumour microenvironment, and Gal-3 inhibitors impaired prostate cancer metastasis in vivo in the Copenhagen rat ${ }^{144}$ or in mouse xenograft models by preventing the interaction between the tumour-associated ThomsenFriedenreich glycoantigen (a cancer-associated glycosylated antigen involved in haematogenous cancer metastasis) and Gal-3 $3^{145}$.

Differential expression of Gal-3 has been detected in biological fluids from patients with prostate cancer and healthy individuals and, therefore, this galectin has been proposed as a biomarker for the early diagnosis of prostate cancer. Gal-3 serum levels in patients with prostate cancer have been reported to be increased in metastatic CSPC or CRPC tumours ${ }^{28}$ or decreased in prostatic adenocarcinomas ${ }^{128,130}$, compared to healthy individuals, whereas Gal-3 levels where reduced in the urine of patients with relapse in comparison to patients without relapse $\mathrm{e}^{130}$. These results have encouraged larger studies to investigate this issue. Indeed, a prospective clinical study that analyzed serum levels of Gal-3 and PSA (and their respective autoantibodies) described positive associations between Gal-3 and PSA levels in 76 men with different stages of prostate cancer (including localized and metastatic disease) and in 19 healthy control individuals ${ }^{146,147}$. Gal3 is an enzyme substrate for MMP-2, MMP-9, and PSA ${ }^{148,149}$, and its cleavage favours tumour progression in human prostate cancer ${ }^{127}$. Finally, Gal-3 has been linked to resistance to enzalutamide and bicalutamide anti-androgen therapy in mouse xenograft models, as its activity increases the levels of the androgen receptor (AR) and AR-target genes ${ }^{136}$, making this galectin a putative therapeutic target for patients with CRPC. Indeed, Gal-3 inhibitors have shown promising results in preclinical studies ${ }^{137,150}$. Interestingly, TFD100, a Gal-3-binding glycopeptide, blocked Gal3-induced T cell apoptosis and impaired angiogenesis and metastasis at nanomolar concentrations in xenograft models ${ }^{137}$. In addition, G3-C12-modified copolymers (targeting Gal-3) improved the anti-tumour activity of 5-Fluorouracil in prostate cancer xenograft mouse models ${ }^{150}$, and modified 
citrus pectin (a natural dietary fiber soluble polysaccharide that functions as an antagonist of extracellular Gal- $3^{151}$ ) sensitized prostate cancer cells to radiotherapy and reduced their migration and invasion capabilites ${ }^{152}$.

Overall, although Gal-3 levels in the tumour are decreased during prostate cancer progression, cytoplasmic overexpression of this galectin has been reported during progression, and in vitro data and preclinical xenograft models have shown that strategies targeting Gal-3 might be effective in impairing prostate cancer progression in vivo.

\section{[H2] Gal-4 in prostate cancer}

Similar to Gal-3, data regarding the levels of Gal-4 in prostate cancer are contradictory. Gal-4 was initially described to be downregulated at the protein level during prostate cancer progression ${ }^{112}$, inferring a potential role for this protein as a tumour suppressor. However, further analyses using prostate cancer tissue microarrays (TMAs) and Oncomine data found a positive correlation between Gal-4 mRNA expression and pathological tumour stage, Gleason score, poor survival outcomes, and biochemical recurrence ${ }^{153}$. In addition, using prostate cancer xenograft mouse models, Gal-4 was shown to promote epithelial-mesenchymal transition (EMT), activation of EGFR and HER2, tumour invasion, and metastasis ${ }^{153}$. In vitro data suggested that Gal-4 can bind to EGFR, HER2, HER3, and IGF1R and trigger their autophosphorylation and activation of downstream pathways and effector proteins, inducing ERK phosphorylation, AKT phosphorylation, fibronectin and TWIST1 expression, and reducing E-cadherin expression ${ }^{153}$. In addition, a 2018 report identified a co-ordinated upregulation of Gal-4 together with aberrant mucin-type $O$-glycosylation in metastatic CRPC, which resulted in a galectin-glycan signalling that led to increased castrationresistance and metastasis in mouse xenograft models and correlated with poor overall survival (OS) in patients ${ }^{154}$.

Thus, although few studies have addressed the implications of Gal-4 expression in prostate cancer, existing data point towards a pro-oncogenic role for this galectin in this pathology.

\section{[H2] Other galectins in prostate cancer}

To date, only one report has presented evidence that Gal-7 is expressed in basal cells in normal prostate tissue and is downregulated in prostate adenocarcinoma ${ }^{155}$. In DU-145 prostate cancer cells, ectopic expression of Gal-7 increased apoptosis induced by etoposide or cisplatin, and Gal-7 showed a tumour suppressive role in prostate cancer as its overexpression resulted in decreased 
in vitro invasion and reduced tumour size in xenograft models ${ }^{155}$. Interestingly, Gal-7 seems to exert a tumour suppressive effect in prostate cancer in both a CRD-dependent and CRDindependent manner; although Gal-7 induces apoptosis in prostate cancer cells independently of its CRD activity, its recognition of glycans via the CRD is required to inhibit in vitro tumour cell invasion and in vivo tumour growth ${ }^{155}$.

Although Gal-8 was initially named prostate carcinoma tumour antigen 1 (PCTA-1) $)^{156,157}$, data regarding its role in prostate cancer are contradictory and nonrobust ${ }^{112,156,158,159}$. Increased Gal-8 mRNA expression was detected in prostate carcinomas compared with normal prostate tissues $^{156}$, and Gal-8 protein levels were found to be upregulated in post-treatment tumour samples $^{159}$, whereas other studies have reported no marked differences in Gal-8 expression between tumour and normal tissues ${ }^{112,158}$. In 2017, in vitro and preclinical models demonstrated that Gal-8 might be involved in tumour metastasis ${ }^{160}$. Interestingly Gal-8 increased E-Cadherin expression and rearranged the cytoskeleton, inhibiting anoikis and promoting tumour cell aggregation, which enabled survival of circulating tumour cells, resulting in metastasis ${ }^{160}$. Immunoglobulin G responses to Gal-3 and Gal-8 increase upon sipuleucel-T treatment in patients with prostate cancer, which correlates with improved overall survival (OS) ${ }^{161}$. Thus, Gal-8 (and Gal3) could conceivably be used as pharmacodynamic predictive biomarkers of response to sipuleucel-T in this pathology.

Despite the importance of Gal-9 in regulating the immune system ${ }^{62,107}$, its presence in prostate cancer has only been analysed in a single descriptive study. Immunohistochemical assessment of prostate tumour tissues from a cohort of 61 patients revealed that Gal-9 was moderately expressed in early-stage prostate cancer compared to benign prostate hyperplasia tissues and gradually decreased with disease progression ${ }^{112}$. Further studies are required to understand the biological effect of Gal-9 downregulation in advanced prostate tumours.

In summary, apart from Gal-1, Gal-3 and Gal-4, the other members of the galectin family have been poorly characterized in prostate cancer development and progression. Gal-7 and Gal-9 levels have been reported to be low in prostate cancer and the former has been characterized as a tumour suppressor in this tumour context. Gal-8 levels have been found to be upregulated and this galectin has been positively associated with metastasis.

\section{[H1] Galectins in bladder cancer}


Galectin expression and the pathological functional effects linked to their dysregulation in bladder cancer have only been partially characterized (TABLE 1, FIG. 2). As in prostate cancer, Gal-1 and Gal-3 have gathered the most attention with in vitro and preclinical studies, whereas other members of the family have only been studied superficially and require further investigation.

\section{[H2] Gal-1 in bladder cancer}

The first study on galectin expression in bladder cancer was published in 1999, in which Gal-1 and Gal-3 mRNA levels were found to be increased in primary or recurrent urothelial carcinomas of the bladder of different grade and stages compared with normal urothelium tissue, with a positive correlation reported between Gal-1 levels and tumour stage and grade ${ }^{162}$. In 2007, Langbein et al. ${ }^{33}$ used a comprehensive qualitative immunohistochemistry approach to characterize six members of the galectin family (Gal-1, Gal-2, Gal-3, Gal-4, Gal-7, and Gal-8) in pTa-pT4 urothelial bladder carcinomas ( $n=61)$. In this study, protein levels of Gal-2, Gal-3, and Gal-8 positively correlated with pathological tumour (pT) stage, and could discriminate between NMIBCs and MIBCs. Moreover, levels of Gal-2 and Gal-8 positively correlated with 5-year disease-specific mortality, and levels of Gal-1, Gal-2, and Gal-8 positively correlated with tumour grade. For Gal-1, although statistical significance was not reached, its immunostaining increased with advancing $T$ stage $^{33}$. In 2015, an extensive study of 185 patients with primary localized bladder cancer (with different disease stages) identified that $>75 \%$ of tumour specimens harboured LGALS1 amplification $^{31}$. These results identified Gal-1 expression as a putative independent prognostic factor for bladder cancer, given that Gal-1 expression could predict disease-specific survival ${ }^{31}$. In addition, Gal-1 expression positively correlated with pT classification, histological grade, vascular invasion, and nodal status ${ }^{31}$. Accordingly, the development of Gal-1-based assays for bladder cancer detection for clinical use is now of particular interest, and the first prototypes have been developed and assessed using bladder cancer cell extracts ${ }^{163}$.

Further research is required to understand the molecular mechanisms triggered by Gal-1 in bladder cancer, but a few studies have offered some insight. Shen et al. ${ }^{164}$ knocked down LGALS1 in two bladder cancer cell lines and found that matrix metalloproteinase 9 (MMP-9) and the RAS-Ras-related C3 botulinum toxin substrate 1 (RAC1)-MEK kinase 4 (MEKK4)-JUN Nterminal kinase (JNK)-activator protein 1 (AP1) signalling pathway mediated the positive effects of Gal-1 on promotion of proliferation, invasion, and clonogenicity. Furthermore, proteomic studies highlighted that shRNA-mediated Gal-1 downregulation in the T24 cell line resulted in 
deregulation of proteins related to lipid and amino acid energy metabolism, the cytoskeleton, cell proliferation, cell-cell interactions, cell apoptosis, metastasis, and protein degradation ${ }^{165}$.

In summary, Gal-1 has been reported to have a relevant role in bladder cancer, in which its expression levels have been found to be prognostic. Further functional validation will deepen the mechanistic insights underpinning the putative pathological effects of Gal-1 in bladder cancer.

\section{[H2] Gal-3 in bladder cancer}

As mentioned previously, Gal-3 mRNA levels were found to be upregulated in bladder cancer ${ }^{162}$ and Gal-3 protein expression levels correlated with $\mathrm{pT}$ stage $\mathrm{e}^{33}$ and were substantially increased in MIBC compared with NMIBC. Gal-3 protein levels could not distinguish between superficial pTa and pT1 bladder cancer ${ }^{32}$, but Gal-3 was identified as an independent negative prognostic marker that predicts recurrence in pTa tumours ${ }^{32}$. A more comprehensive study showed overexpression of Gal-3 at the mRNA and protein levels in a large cohort of patients with bladder cancer, positively correlating Gal-3 expression with tumour stage, grade, and overall survival (OS) in patients with T1 Grade 3 (T1G3) tumours ${ }^{26}$. Moreover, Gal-3 expression levels could discriminate between MIBC and NMIBC tumours ${ }^{26}$. Interestingly, modified citrus pectin (a Gal-3 antagonist ${ }^{151}$ ) inhibited cell viability in T24 and $\mathrm{J} 82$ human bladder cancer cell lines through cell cycle arrest and caspase-3 activation, and impaired tumour growth when administered orally in xenograft models ${ }^{166}$.

Importantly, soluble Gal-3 detection in urine ${ }^{26}$ and serum ${ }^{27}$ has been proposed as a tool for bladder cancer diagnosis, as Gal-3 urinary and serum protein levels were found to discriminate patients with bladder cancer from control individuals ${ }^{26,27}$.

In summary, Gal-3 has been reported to be overexpressed in bladder cancer and its expression is positively regulated during disease progression, suggesting the utility of Gal-3 as a diagnostic biomarker. However, additional in vitro and preclinical studies are needed to fully understand the molecular mechanisms triggered by Gal-3 in this pathology.

\section{[H2] Other galectins in bladder cancer}

Very few studies have addressed the involvement of the other galectins in bladder cancer. Gal-4 downregulation by hypermethylation of its promoter positively correlated with histological grade, pT stage, lymph node metastasis, and poor prognosis in patients with different stages (including non-recurrent or recurrent, early or advanced stages) of NMIBC and $\mathrm{MIBC}^{34}$. In addition, in vitro data have further demonstrated that Gal-4 functions as a tumour suppressor in bladder cancer cell 
lines by inhibiting cell proliferation, migration, and invasion ${ }^{34}$. Similarly, Gal-7 expression is reduced in bladder cancer compared with normal urothelium ${ }^{33,167}$, suggesting it has a role in inhibiting tumour growth (comparable to its putative role in prostate cancer ${ }^{155}$ ). Indeed, also analogous to prostate cancer, Gal-7 overexpression in bladder cancer cell lines also increased sensitivity to chemotherapy, and chemosensitive-patients showed higher Gal-7 levels than those who exhibited resistance to cisplatin chemotherapy ${ }^{167}$. Loss of Gal-8 expression has also been reported as an early step in the development of bladder cancer, and Gal-8 downregulation was also associated with tumour recurrence ${ }^{168}$. Finally, high Gal-9 expression levels in bladder tumours correlated with improved patient survival, although chemotherapy was more effective in patients whose tumours had low Gal-9 expression, suggesting that this Gal-9 might be useful as a predictive biomarker for therapy selection ${ }^{35}$.

Thus, although few articles have described the role of Gal-4, Gal-7, Gal-8 and Gal-9 in bladder cancer, most of these reports found downregulation of these galectin family members, suggesting their tumour suppressor activities in bladder cancer.

\section{[H1] Opportunities for clinical translation}

The potential of galectins in the diagnosis and management of prostate cancer and bladder cancer is evident, but translation of this basic knowledge into the clinic is in its infancy.

Given the role of several galectins in tumour angiogenesis and immune evasion, the clinical opportunities offered by the galectin family in the field of cancer could extend beyond the direct therapeutic targeting of galectins to include patient risk stratification and treatment selection in the era of personalized medicine. In this regard, galectins - particularly Gal-1, Gal-3, Gal-8, and Gal-9 - have been described as master governors of the angiogenic cascade in cancer, as they control endothelial cell activation, proliferation, adhesion, migration, tube formation, and sprouting, ultimately driving tumour progression in preclinical studies ${ }^{169}$. Moreover, several members of the galectin family have been described as key regulators of the tumour immune response in several cancer settings. For instance, Gal-1 impairs transendothelial migration of T cells and natural killer (NK) cell recruitment and induces apoptosis in $\mathrm{CD}^{+}{ }^{+}$helper $1\left(\mathrm{~T}_{H} 1\right)$ and $\mathrm{T}_{\mathrm{H}} 17$ cells and $\mathrm{CD} 8^{+}$cytotoxic $\mathrm{T}$ cells, differentiation of regulatory $\mathrm{T}$ cells ( $\mathrm{T}_{\text {regs }}$ ) and dendritic cells (DCs), M2 macrophage polarization, and expansion of myeloid-derived suppressor cells (MDSCs), resulting in profound tumour immune evasion ${ }^{104,105,107,170}$. Similarly, Gal-3 can also hamper the antitumour immune response by inducing $T$ cell anergy, impairing $T$ cell activation, and inhibiting 
NK cell function ${ }^{107,171,172}$. Moreover, Gal-9 triggers specific apoptosis in CD8 ${ }^{+}$cytotoxic T cells, $\mathrm{T}_{\text {reg }}$ differentiation, and expansion of $\mathrm{MDSCs}^{173-175}$. These data reveal several opportunities for translation of altered galectin expression levels in cancer to clinical management, which could be extrapolated to prostate or bladder cancer, given the changes in galectin expression patterns that have been reported in these diseases (TABLE 1). First, the presence of high levels of proangiogenic or immunosuppressive galectins in a tumour might predict poor response to antiangiogenic therapies or immunotherapies, respectively, and, therefore, could be a useful predictive biomarker for patient stratification. Second, targeting these galectins could have multiple therapeutic effects, including blockade of tumour proliferation and angiogenesis and restoration of the antitumour immune response. Last, but not least, the combination of galectin inhibitors with standard chemotherapy, radiotherapy, antiangiogenic drugs, or immunotherapy agents could improve treatment efficacy and patient response to therapy.

Interestingly, therapeutic targeting of Gal-3 with pectin (which functions as an antagonist of extracellular Gal-3 ${ }^{151}$ ) has been reported to reduce prostate cancer cell migration and invasion and sensitize prostate cancer cells to radiotherapy ${ }^{152}$, decreases cell viability in bladder cancer cell lines and reduces tumour growth in bladder cancer xenograft models ${ }^{166}$. Other Gal-3 inhibitors have been reported to induce $T$ cell apoptosis, impair angiogenesis and metastasis ${ }^{137}$, and improve the efficacy of 5-Fluorouracil chemotherapy ${ }^{150}$ in prostate cancer xenograft models. Moreover, preclinical data with both AR-positive and AR-negative prostate cancer xenograft models have indicated that targeting Gal-1 using a small molecule Gal-1 inhibitor (LLS30) inhibits tumour progression and metastasis in prostate cancer, and potentiates the antitumour effects of docetaxel to achieve complete tumour regression ${ }^{121}$. The small molecule Gal-1 inhibitor OTX008 has been tested in a phase I clinical trial ${ }^{176}$ as a single agent for the treatment of patients with advanced solid tumours, but no objective responses were observed ${ }^{177}$. The natural polysaccharide GM-CT-01 (galactomannan, also called Davanat ${ }^{\circledR}$ ), which targets both Gal-1 and Gal-3, was reported to be well tolerated in a phase I clinical trial in combination with 5-Fluorouracil chemotherapy ${ }^{178,179}$ for the treatment of different tumour types, including one patient with prostate cancer ${ }^{180}$. GM-CT-01 was also tested together with 5-Fluorouracil in a phase II clinical trial in patients with colorectal cancer ${ }^{180}$ and in a phase $\mathrm{I} / \mathrm{II}$ trial in combination with peptide vaccines in patients with melanoma ${ }^{181}$. Inhibition of Gal-3 with GR-MD-02, a polysaccharide polymer with high affinity for Gal-3, increased survival in the TRAMP-C1 prostate cancer cell line model ${ }^{182}$ and this agent has also been tested in combination with immunotherapy in ongoing clinical trials designed 
for melanoma, non-small cell lung cancer and squamous cell carcinoma of the head and neck ${ }^{183,184}$. Despite the robust rationale from preclinical data on targeting galectins in bladder cancer, no clinical trials based on galectin inhibitors have yet been performed in bladder cancer.

The observation that specific galectin members with putative tumour suppressor functions - such as Gal-7 ${ }^{155}$ and Gal-9 ${ }^{112}$ in prostate cancer, or Gal-4 ${ }^{34}$, Gal- $7^{33,167}$, Gal-8 $8^{168}$ and Gal-9 $9^{35}$ in bladder cancer - are downregulated in prostate cancer and bladder cancer (TABLE 1) also presents the opportunity of using recombinant galectins, galectin mimetic compounds, pharmacological agonists or gene transfer for cancer therapy. For instance, the finding that patients with bladder cancers whose tumours have high levels of Gal-7 have improved response to chemotherapy ${ }^{167}$ suggests that one novel approach for treating patients with chemoresistant bladder cancer could be to induce Gal-7 expression in their tumours. Remarkably, these strategies (for example, induction of Gal-1, Gal-2 or Gal-3 expression) have shown promising results in preclinical models of inflammatory disorders ${ }^{185-187}$.

Galectins have also been considered as predictive biomarkers for patient risk stratification and treatment selection. Gal-7 levels has been shown to be higher in cisplatin-sensitive patients with urothelial cancer than in chemoresistant patients ${ }^{167}$, indicating that detection of Gal-7 in bladder cancer biopsy specimens before treatment - or ideally with noninvasive techniques such as serum detection - might be used as a predictive biomarker for chemotherapy response ${ }^{167}$. Similarly, cisplatin-based adjuvant chemotherapy was more effective in low Gal-9-expressing bladder tumours than in those with high Gal-9 expression ${ }^{35}$. Gal-3 was also identified as a marker for basal features in the prostate cancer epithelium, highlighting its potential to distinguish between tumour subtypes and enhance therapeutic efficiency with personalized medicine ${ }^{29}$.

Galectins could also aid in the diagnosis of patients with prostate cancer or bladder cancer. Owing to galectin secretion outside of the cell, detectable levels of these proteins can be determined in peripheral blood urine and semen $^{79-81}$. Several studies have already shown the diagnostic potential of determining galectin levels in the sera of patients with other cancers ${ }^{81,188-}$ 192. Detection of galectin levels in blood would be useful test to complement serum PSA testing in the diagnosis, active surveillance and follow-up monitoring of patients with prostate cancer ${ }^{28-}$ $30,146,147$. For instance, Gal-3 serum levels are substantially increased in patients with metastatic prostate cancer compared with healthy individuals ${ }^{28}$ and Gal-3 levels are positively associated with PSA levels, particularly at early clinical presentation ${ }^{146,147}$. In addition, a diagnostic sensitive assay based on methylation-specific PCR was developed for stage I and II prostate cancer ${ }^{30}$. A link 
between Gal-3 levels and bladder cancer has been described, detecting with increased Gal-3 levels having been detected in serum from patients with bladder cancer compared to controls, but no studies have addressed other members of the galectin family $27,193,194$.

Studies describing altered patterns of galectin expression in prostate or bladder cancer and the identification of their functional effects in preclinical models have provided opportunities for clinical translation. In line with the fact that the best studied members of the galectin family Gal-1 and Gal-3 - are found to be overexpressed in cancer and promote tumour development and progression, the first clinical trials evaluating inhibition of these galectins in combination with approved therapies have been performed, although prostate or bladder cancer have not yet been included. Importanty, galectins have not only been considered as therapeutic targets, and they could also aid in diagnosis, patient risk stratification, and treatment selection in prostate cancer and bladder cancer.

\section{[H1] Future challenges}

The human galectin family comprises 11 proteins that have pleiotropic functions in cancer. The precise pattern of galectin expression and the particular tumour microenvironment need to be considered in order to successfully design galectin-based pharmacologic modulators. Improved preclinical models will be essential to deepen our understanding of the functional effects of galectin dysregulation and therapeutic galectin modulation before moving to clinical trials.

\section{[H2] Galectin redundancy and therapeutic implications}

Altered galectin expression and aberrant patterns of protein glycosylation in cancer cells - which, in turn, can modify galectin recognition and cause further downstream signalling effects - occur in prostate and bladder cancers. Improved understanding of the extreme complexity of galectins and aberrant glycosylation in cancer cells will open new avenues for improving diagnosis and therapeutic interventions. Increasing data have reported changes in galectin expression levels in prostate and bladder cancer, although many conflicting reports obscure the protumorigenic and/or antitumorigenic roles of different family members in these diseases (TABLE 1). Several issues, such as cell localization, tissue compartment, redox status, and post-translational modifications, must be considered to improve the understanding of the role of galectins. Considering the key role of several galectins (for example, Gal-1, Gal-3, and Gal-9) in angiogenesis and tumour immune evasion ${ }^{62}$, the development of pharmacological modulators targeting 
galectin-glycan interactions is an emerging field in cancer therapy. Nevertheless, as the CRD is highly conserved among galectin family members, special attention must be paid when using galectin pharmacological modulators targeting this domain, as low selectivity could hamper the benefits of blocking a specific galectin in a specific context. Importantly, results from galectin knockout mice have revealed that several members of the family can have overlapping activities and, therefore, can functionally compensate for each other. For instance, LGALS1, LGALS3, and even double LGALS1-LGALS3 knockout mice are viable and fertile ${ }^{108,195}$; however, mice deficient in either one of these genes ${ }^{196,197}$ show different phenotypes when challenged in different experimental settings, particularly regarding immune responses and inflammation (including cancer), indicating that each galectin member has functional uniqueness ${ }^{94}$. The fact that simple galectin-knockout mice do not show overall phenotypic effects suggest that the physiological roles of galectins could possibly be compensated by other members of the family, which could be interesting when considering individual galectins as therapeutic targets and the possible adverse effects. However, functional redundancy also complicates clinical strategies and makes it essential to obtain a deep understanding of the galectin expression profile, not only in every tumour type but also in every particular patient before translating this information into clinical practice. Some galectins can have a dual role depending on the unique cellular environment and conditions and, therefore, pharmacological modulation could tilt the balance towards an antitumorigenic or protumorigenic response, so special caution is required. The fact that some of the galectintargeted agents in clinical trials can bind different members of the galectin family with different affinities further complicates the interpretation of their results. In this regard, specific anti-galectin blocking antibodies could offer higher selectivity than the glycan-based inhibitors ${ }^{45,198,199}$. Overall, the complexity of galectin binding with different ligands in different microenvironments, which, in turn, modifies galectin affinity for certain ligands, challenges the design of pharmacological modulators.

\section{[H2] Preclinical modelling}

Robust data have demonstrated the relevance of galectins in vitro, but few reports have addressed the in vivo effects of glycan-galectin interactions on genitourinary tumour development and progression. Most in vivo studies have used xenograft models despite their limitations, which include poor modelling of tumor-stromal interactions, the lack of a functional immune system, and the fact that they cannot model tumorigenesis and, therefore, lead to tumours that fail to 
recapitulate heterogeneity, genetics and histology of human tumours ${ }^{200,201}$, hindering clinical translation. Considering the relevance of several galectin members in the stroma and in regulation of metastasis and immune evasion, the limitations of xenograft models highlight the need to move towards transgenic animal models that faithfully recapitulate tumour progression and the metastatic cascade, as well as the immune microenvironment ${ }^{200}$, in order to study the galectin family of proteins in prostate and bladder cancer pathology. Although several prostate cancer 202,203 and bladder cancer ${ }^{204}$ genetically-engineered mouse (GEM) models have been developed, to date, none have been used to study the galectin family of proteins.

Patient-derived xenografts (PDX) models and patient-derived organoids ${ }^{205}$ have also been described for prostate $206-208$ and bladder cancer ${ }^{209-211}$, and their use enables investigators to recapitulate the unique genomic and molecular properties of individual human tumours, test drug responses, and develop personalized medicine strategies. These models might be an important tool to complement animal models for the study of the galectin-glycan axis in prostate and bladder cancer, and for analysis the preclinical effects of galectin modulators. However, tissue availability (for prostate cancer), impaired tumor-stroma interactions, and lack of a functional immune system might limit the use of human organoids and PDX to study the effects of galectins or galectin pharmacological modulators in prostate and bladder tumours.

\section{[H1] Conclusions}

The development of effective therapies for patients with prostate cancer and bladder cancer, particularly for those with advanced-stage disease, is an unmet clinical need that requires new therapeutic molecular targets. Most treatments currently target tumour cells, an approach that we now know underestimates the importance of the tumour microenvironment during tumour progression and metastatic dissemination. Although new clinical trials evaluating $\mathrm{ICI}$ immunotherapy have shown positive results for patients with bladder cancer ${ }^{15,16}$, only a subset of patients respond to this treatment, highlighting the importance of predictive biomarkers for patient stratification and the identification of new immunotherapies or combinations with improved efficacy. Clinical studies have also been performed with immunotherapies in prostate cancer, although ICls have shown limited success $^{19}$, potentially due to the highly immunosuppressive microenvironment of prostate cancer. Only sipuleucel-T has shown clinical efficacy in prostate cancer, although major drawbacks have hampered its incorporation into clinical practice ${ }^{212}$, and patients with metastatic CRPC, despite improvements (for example, next- 
generation AR pathway inhibitors, such as enzalutamide or abiraterone acetate, or new taxanes such as cabazitaxe $\left.{ }^{6}\right)$, still have limited survival outcomes ${ }^{212-215}$.

Most of the galectins studied so far have shown altered expression patterns in prostate and bladder cancers, and preclinical data have shed some light on the functional roles of galectins in the tumour context. In prostate cancer, compared with normal prostate tissues, Gal-1 has been reported to be overexpressed ${ }^{112,116,117}, \mathrm{Gal}^{-7^{155}}$ and Gal-9 $9^{112}$ downregulated, whereas controversial data have been described for Gal-3 $30,112,114,115,125-131$, Gal-4 ${ }^{112,153}$ and Gal-8 $8^{112,156,158,159}$. In bladder cancer, Gal-1 and Gal-3 have also been found to be overexpressed $31,33,162,26$, whereas Gal-4 ${ }^{34}$, Gal$7^{33,167}$ and Gal-8 ${ }^{168}$ were reported to be downregulated. Using in vitro and in vivo data obtained from cell line and xenograft models, galectins have been implicated in a wide variety of cancerdriving events in prostate and bladder cancer, such as angiogenesis, T cell apoptosis, migration, proliferation, and metastasis, among others, opening the door to strategies modulating galectin activity as new avenues for therapy.

Despite the strong potential of the galectin family for the development of diagnostics and effective new treatments for bladder and prostate cancer, several important challenges should be addressed before galectin-based strategies can be translated to clinical settings. First, a comprehensive understanding of the link between the overall tissue galectin profile and altered tumour glycosylation in the context of bladder and prostate tumours is needed to inform the rationale and flaws of galectin targeting strategies. Second, the identification of the specific or redundant functions (protumorigenic or antitumorigenic roles) of different galectins in the context of prostate cancer and bladder cancer could help to predict the effect of galectin modulators. Third, the development of galectin-glycan inhibitors with well-understood specificity (that is, if they target one or several members) is necessary to understand and predict therapeutic effects. Last, validation of galectin antagonists (or agonists, for those galectins with antitumour functions) using preclinical model systems that faithfully mimic prostate and bladder tumour development and progression (for example, transgenic mouse models and patient-derived organoids) will be necessary to investigate the therapeutic potential of galectin-targeting strategies before clinical translation. Studies aimed at overcoming these challenges regarding the use of galectins in the clinical management of patients with prostate cancer and bladder cancer are now required.

Neus Martínez-Bosch ${ }^{1,2 *}$, Alejo Rodriguez-Vida ${ }^{1,3}$, Núria Juanpere ${ }^{4}$, Josep Lloreta ${ }^{4}$, Ana Rovira ${ }^{1}$, Joan Albanell ${ }^{1,3}$, Joaquim Bellmunt ${ }^{1,5}$, and Pilar Navarro ${ }^{1,2,6 *}$ 
${ }^{1}$ Cancer Research Program, Hospital del Mar Medical Research Institute (IMIM), Unidad Asociada CSIC, Barcelona, Spain.

${ }^{2}$ Unidad Asociada I+D+i IMIM-Institute of Biomedical Research of Barcelona, IIBB (CSIC), Barcelona, Spain.

${ }^{3}$ Department of Medical Oncology, Hospital del Mar, Barcelona, Spain. ${ }^{4}$ Department of Pathology, Hospital del Mar, Barcelona, Spain. ${ }^{5}$ Bladder Cancer Center, Dana-Farber Cancer Institute/Brigham and Women's Cancer Center, Harvard Medical School, Boston, MA , USA. ${ }^{6}$ Institute of Biomedical Research of Barcelona (IIBB-CSIC), Barcelona, Spain. 


\section{Acknowledgements}

This work was supported by grants from the Spanish Ministry of Economy and Competitiveness/ ISCIII-FEDER (PI14/00125; PI17/00199), AECC-Cataluña 2015 grant, and the Generalitat de Catalunya (2014/SGR/143 and 2017/SGR/225) grant to P.N. The authors thank V.A. Raker for valuable comments and input on language.

\section{Author Contributions}

All authors researched data for the article, made substantial contributions to discussion of the article contents, and reviewed and/or edited the manuscript before submission. P.N. and N. M.-B. wrote the manuscript.

\section{Competing interests}

The authors declare no competing interests.

\section{Publisher's note}

Springer Nature remains neutral with regard to jurisdictional claims in published maps and institutional affiliations. 
-ONLINE ONLY-

\section{Subject categories}

Health sciences / Urology / Urogenital diseases / Urological cancer / Prostate cancer [URI /692/4025/2768/589/466]

Health sciences / Urology / Urogenital diseases / Urological cancer / Bladder cancer [URI /692/4025/2768/589/1336]

Biological sciences / Cell biology / Post-translational modifications / Glycosylation [URI /631/80/458/1524]

Health sciences / Medical research / Preclinical research [URI /692/308/2778]

\section{Table of contents blurb}

Galectins have established roles in cancer, but their function in genitourinary cancer is unclear. In this Opinion, the authors discuss the emerging roles of galectins in prostate and bladder cancer development and progression, and speculate regarding opportunities for clinical translation. 


\section{Figure 1 | Classification of the mammalian galectins.}

The 15 mammalian galectins (asterisks denote the 11 galectins found in humans) are classified into three subfamilies on the basis of their structure, specifically the number and organization of their carbohydrate recognition domains (CRDs) ${ }^{63}$. The prototype galectins contain a single CRD and are either present as monomers (Gal-5, Gal-10, and Gal-14) or dimers held together by a hydrophobic core (Gal-1, Gal-2, Gal-7, Gal-11, and Gal-15) or disulfide bridges (Gal-13; indicated as two dots). The tandem-repeat galectins (Gal-4, Gal-6, Gal-8, Gal-9, and Gal-12) have two CRDs linked by a short linker peptide. Gal-3, the only chimeric-type galectin, has a single CRD and a large aminoterminal domain that enables oligomerization. The CRD is structurally and evolutionary conserved in all galectin family members (represented here as a crescent). 


\section{Figure 2 | Roles of galectins in prostate and bladder tumour development and progression.}

The schematic depicts the most important known functional roles for each galectin family member in prostate cancer (blue boxes), bladder cancer (yellow box), or in both (green boxes). In prostate cancer, preclinical data have established a role for galectins in driving many important events that trigger tumour development and progression. Gal-1 has been reported to mediate tumour cell adhesion to the extracellular matrix (ECM) in prostate cancer ${ }^{115}$. In addition, Gal-3 and Gal-8 are involved in tumour cell homotypic aggregation ${ }^{145,160}$, whereas Gal-1, Gal-3, Gal-4 and Gal-7 are involved in tumour cell migration and/or invasion 121,133,136,140,145,153, Gal-4 mediates epithelialmesenchymal transition (EMT) in prostate tumour cells ${ }^{153}$ and, together with Gal-1, Gal-3, and Gal8, regulates metastasis ${ }^{29,121,137,142-145,153,154,160}$. Gal-1, Gal-3, Gal-7 and Gal-8 also regulate prostate tumour cell apoptosis ${ }^{121,29,133,155}$. Tumour cell-endothelial cell (heterotypic) aggregation and angiogenesis have been shown to be induced by Gal-1 and Gal-3 ${ }^{116,137-139,144,145}$, Gal-1 and Gal-3 promote an immune suppressive state by inducing $T$ cell apoptosis and by Gal-1-dependent inhibition of $T$ cell proliferation, cytotoxic activation and extravasation to the endothelium ${ }^{118,119,124,137}$. Finally, Gal-1, Gal-3 and Gal-7 mediate tumour cell proliferation and tumour growth in prostate cancer ${ }^{121,127,133,136,155}$. In bladder cancer, galectin-driven functional phenotypes are much less well established than in prostate cancer. In this pathology, Gal-1 increases tumour cell proliferation and invasion ${ }^{164}$, Gal-3 regulates tumour cell viability and tumour growth ${ }^{166}$, and Gal-4 inhibits tumour growth, migration and invasion ${ }^{34}$. 
Table 1 | Galectin expression and phenotypic effects in prostate and bladder cancer.

\begin{tabular}{|c|c|c|c|}
\hline Galectin & Expression & Phenotypic effect & Refs \\
\hline \multicolumn{4}{|c|}{ Prostate cancer } \\
\hline Gal-1 & Upregulated & $\begin{array}{ll}\text { - } & \text { Induces adhesion of tumour cells to ECM } \\
\text { - } & \text { Induces tumour cell-EC aggregation } \\
\text { - Increases angiogenesis in vitro and in xenograft } \\
\text { models } \\
\text { Inhibits T cell extravasation across endothelial cells } \\
\text { in vitro } \\
\text { - Induces T cell apoptosis and reduces its } \\
\text { - } \quad \text { Induces tumour cell migration and invasion in vitro } \\
\text { - Induces metastasis in xenograft models } \\
\text { - Induces tumour cell proliferation in vitro and in } \\
\text { xenograft models } \\
\text { Decreases the antitumour effects of docetaxel in } \\
\text { vitro and in xenograft models }\end{array}$ & $\begin{array}{l}115 \\
116 \\
112 \\
118 \\
119,124 \\
121 \\
121 \\
121 \\
121\end{array}$ \\
\hline \multirow[t]{2}{*}{ Gal-3 } & Upregulated & $\begin{array}{l}\text { - Induces tumour cell migration in vitro } \\
\text { - Induces tumour cell invasion in vitro } \\
\text { - Induces cell proliferation in vitro and in xenograft } \\
\text { - } \quad \text { Induces adhesion of tumour cells to endothelial } \\
\text { cells in vitro } \\
\text { - } \quad \text { Induces tumour cell AIG in vitro } \\
\text { - Induces angiogenesis in vitro and in vivo } \\
\text { - Induces metastasis in xenograft models and in the } \\
\text { - } \quad \text { Induces T cell apoptosis in vitro } \\
\text { - Promotes drug resistance in vitro and in xenograft } \\
\text { models }\end{array}$ & $\begin{array}{l}136,140,145 \\
133,136 \\
127,133,136, \\
137,138,139,144,145 \\
29,133 \\
127,133,136,144,145 \\
133,137 \\
29,137,142,143,144,145 \\
137 \\
134,136,135,150,152\end{array}$ \\
\hline & Downregulated & $\begin{array}{l}\text { - Nuclear Gal-3 reduces invasion, tumour cell } \\
\text { apoptosis, and anchorage-independent growth in } \\
\text { vitro } \\
\text { - Nuclear Gal-3 reduces tumour growth and } \\
\text { angiogenesis in vivo (xenograft models) }\end{array}$ & 133 \\
\hline \multirow[t]{2}{*}{ Gal-4 } & Upregulated & $\begin{array}{l}\text { Promotes EMT, tumour invasion and metastasis in } \\
\text { xenograft model }\end{array}$ & 153,154 \\
\hline & Downregulated & Unknown & 112 \\
\hline Gal-7 & Downregulated & $\begin{array}{ll}\text { - } & \text { Decreases invasion in vitro } \\
\text { - } & \text { Reduces tumour growth in xenograft models } \\
\text { - } & \text { Induces tumour cell apoptosis }\end{array}$ & $\begin{array}{l}155 \\
155 \\
155\end{array}$ \\
\hline \multirow[t]{2}{*}{ Gal-8 } & Upregulated & Promotes metastasis & 160 \\
\hline & Unchanged & Unknown & 112,158 \\
\hline \begin{tabular}{l|l} 
Gal-9 & \\
\end{tabular} & Downregulated & Unknown & 112 \\
\hline \multicolumn{4}{|c|}{ Bladder cancer } \\
\hline Gal-1 & Upregulated & $\begin{array}{l}\text { Increases proliferation, invasion and clonogenicity in } \\
\text { vitro }\end{array}$ & 164 \\
\hline Gal-3 & Upregulated & $\begin{array}{ll}\text { - } & \text { Increases tumour cell viability in vitro } \\
\text { - } & \text { Induces tumour growth in xenograft models } \\
\end{array}$ & $\begin{array}{l}166 \\
166 \\
\end{array}$ \\
\hline Gal-4 & Downregulated & Inhibits cell proliferation, migration and invasion in vitro & 34 \\
\hline Gal-7 & Downregulated & Increases sensitivity to chemotherapy & 167 \\
\hline Gal-8 & Downregulated & Unknown & 168 \\
\hline
\end{tabular}


ECM, extracellular matrix; EMT, epithelial-mesenchymal transition; EC, endothelial cell 



\section{References}

1. Siegel, R. L., Miller, K. D. \& Jemal, A. Cancer statistics, 2018. CA. Cancer J. Clin. 68, 7-30 (2018).

2. Risbridger, G. P., Toivanen, R. \& Taylor, R. A. Preclinical Models of Prostate Cancer: PatientDerived Xenografts, Organoids, and Other Explant Models. Cold Spring Harb. Perspect. Med. 8, a030536 (2018).

3. Fizazi, K. et al. Abiraterone plus Prednisone in Metastatic, Castration-Sensitive Prostate Cancer. N. Engl. J. Med. 377, 352-360 (2017).

4. James, N. D. et al. Abiraterone for Prostate Cancer Not Previously Treated with Hormone Therapy. N. Engl. J. Med. 377, 338-351 (2017).

5. Shipley, W. U. et al. Radiation with or without Antiandrogen Therapy in Recurrent Prostate Cancer. N. Engl. J. Med. 376, 417-428 (2017).

6. Sathianathen, N. J., Konety, B. R., Crook, J., Saad, F. \& Lawrentschuk, N. Landmarks in prostate cancer. Nat. Rev. Urol. 15, 627-642 (2018).

7. Cotter, K. A. \& Rubin, M. A. Sequence of events in prostate cancer. Nature 560, 557-559 (2018).

8. Arriaga, J. M. \& Abate-Shen, C. Genetically Engineered Mouse Models of Prostate Cancer in the Postgenomic Era. Cold Spring Harb. Perspect. Med. 9, a030528 (2019).

9. van der Toom, E. E. et al. Prostate-specific markers to identify rare prostate cancer cells in liquid biopsies. Nat. Rev. Urol. 16, 7-22 (2019).

10. Sved, P. D., Gomez, P., Manoharan, M., Kim, S. S. \& Soloway, M. S. Limitations of biopsy Gleason grade: implications for counseling patients with biopsy Gleason score 6 prostate cancer. J. Urol. 172, 98-102 (2004).

11. Cohen, M. S. et al. Comparing the Gleason Prostate Biopsy and Gleason Prostatectomy Grading System: The Lahey Clinic Medical Center Experience and an International MetaAnalysis. Eur. Urol. 54, 371-381 (2008).

12. Baciarello, G., Gizzi, M. \& Fizazi, K. Advancing therapies in metastatic castration-resistant prostate cancer. Expert Opin. Pharmacother. 19, 1797-1804 (2018).

13. Xu, J. X. et al. FDA Approval Summary: Nivolumab in Advanced Renal Cell Carcinoma After Anti-Angiogenic Therapy and Exploratory Predictive Biomarker Analysis. Oncologist 22, 311-317 (2017).

14. Powles, T. et al. Updated European Association of Urology Guidelines Recommendations 
for the Treatment of First-line Metastatic Clear Cell Renal Cancer. Eur. Urol. 73, 311-315 (2017).

15. Bellmunt, J. et al. Pembrolizumab as Second-Line Therapy for Advanced Urothelial Carcinoma. N. Engl. J. Med. 376, 1015-1026 (2017).

16. Balar, A. V et al. First-line pembrolizumab in cisplatin-ineligible patients with locally advanced and unresectable or metastatic urothelial cancer (KEYNOTE-052): a multicentre, single-arm, phase 2 study. Lancet Oncol. 18, 1483-1492 (2017).

17. Siefker-Radtke, A. \& Curti, B. Immunotherapy in metastatic urothelial carcinoma: focus on immune checkpoint inhibition. Nat. Rev. Urol. 15, 112-124 (2017).

18. Carlo, M. I., Voss, M. H. \& Motzer, R. J. Checkpoint inhibitors and other novel immunotherapies for advanced renal cell carcinoma. Nat. Rev. Urol. 13, 420-431 (2016).

19. Maia, M. C. \& Hansen, A. R. A comprehensive review of immunotherapies in prostate cancer. Crit. Rev. Oncol. Hematol. 113, 292-303 (2017).

20. Matsumoto, K. et al. Late Recurrence and Progression in Non-muscle-invasive Bladder Cancers After 5-year Tumor-free Periods. Urology 75, 1385-1390 (2010).

21. Tabayoyong, W. B. et al. Systematic Review on the Utilization of Maintenance Intravesical Chemotherapy in the Management of Non-muscle-invasive Bladder Cancer. Eur. Urol. Focus 4, 512-521 (2018).

22. Türkölmez, K., Tokgöz, H., Reşorlu, B., Köse, K. \& Bedük, Y. Muscle-invasive bladder cancer: predictive factors and prognostic difference between primary and progressive tumors. Urology 70, 477-81 (2007).

23. Marcq, G. et al. Contemporary best practice in the use of neoadjuvant chemotherapy in muscle-invasive bladder cancer. Ther. Adv. Urol. 11, 175628721882367 (2019).

24. Rodriguez-Vida, A., Perez-Gracia, J. L. \& Bellmunt, J. Immunotherapy Combinations and Sequences in Urothelial Cancer: Facts and Hopes. Clin. Cancer Res. 24, 6115-6124 (2018).

25. Anastasiadis, A. \& de Reijke, T. M. Best practice in the treatment of nonmuscle invasive bladder cancer. Ther. Adv. Urol. 4, 13-32 (2012).

26. Canesin, G. et al. Galectin-3 expression is associated with bladder cancer progression and clinical outcome. Tumour Biol. 31, 277-85 (2010).

27. Sakaki, M. et al. Serum level of galectin-3 in human bladder cancer. J. Med. Invest. 55, 12732 (2008).

28. Balan, V. et al. Galectin-3: a possible complementary marker to the PSA blood test. 
Oncotarget 4, 542-9 (2013).

29. Wang, Y. et al. The significance of galectin-3 as a new basal cell marker in prostate cancer. Cell Death Dis. 4, e753 (2013).

30. Ahmed, H., Cappello, F., Rodolico, V. \& Vasta, G. R. Evidence of heavy methylation in the galectin 3 promoter in early stages of prostate adenocarcinoma: development and validation of a methylated marker for early diagnosis of prostate cancer. Transl. Oncol. 2, 146-56 (2009).

31. Wu, T.-F. et al. Galectin-1 Dysregulation Independently Predicts Disease Specific Survival in Bladder Urothelial Carcinoma. J. Urol. 193, 1002-1008 (2015).

32. Kramer, M. W. et al. Decreased expression of galectin-3 predicts tumour recurrence in $\mathrm{pTa}$ bladder cancer. Oncol. Rep. 20, 1403-8 (2008).

33. Langbein, S. et al. Gene-expression signature of adhesion/growth-regulatory tissue lectins (galectins) in transitional cell cancer and its prognostic relevance. Histopathology 51, 681690 (2007).

34. Wu, M.-M. et al. Promoter hypermethylation of LGALS4 correlates with poor prognosis in patients with urothelial carcinoma. Oncotarget 8, 23787-23802 (2017).

35. Liu, Y. et al. Galectin-9 as a prognostic and predictive biomarker in bladder urothelial carcinoma. Urol. Oncol. Semin. Orig. Investig. 35, 349-355 (2017).

36. Cummings, R. D., Liu, F.-T. \& Vasta, G. R. Galectins. Essentials of Glycobiology (2015).

37. Tsai, C.-M. et al. Galectin-1 and galectin-8 have redundant roles in promoting plasma cell formation. J. Immunol. 187, 1643-52 (2011).

38. Tribulatti, M. V., Figini, M. G., Carabelli, J., Cattaneo, V. \& Campetella, O. Redundant and antagonistic functions of galectin-1, -3 , and -8 in the elicitation of T cell responses. $J$. Immunol. 188, 2991-9 (2012).

39. Vyakarnam, A., Dagher, S. F., Wang, J. L. \& Patterson, R. J. Evidence for a role for galectin-1 in pre-mRNA splicing. Mol.Cell Biol. 17, 4730-4737 (1997).

40. Munkley, J., Mills, I. G. \& Elliott, D. J. The role of glycans in the development and progression of prostate cancer. Nat. Rev. Urol. 13, 324-333 (2016).

41. Ohyama, C. Glycosylation in bladder cancer. Int. J. Clin. Oncol. 13, 308-313 (2008).

42. Stowell, S. R., Ju, T. \& Cummings, R. D. Protein glycosylation in cancer. Annu. Rev. Pathol. 10, 473-510 (2015).

43. Pinho, S. S. \& Reis, C. A. Glycosylation in cancer: mechanisms and clinical implications. Nat. 
Rev. Cancer 15, 540-55 (2015).

44. Rabinovich, G. A. \& Croci, D. O. Regulatory circuits mediated by lectin-glycan interactions in autoimmunity and cancer. Immunity. 36, 322-335 (2012).

45. Croci, D. O. et al. Glycosylation-dependent lectin-receptor interactions preserve angiogenesis in anti-VEGF refractory tumors. Cell 156, 744-58 (2014).

46. Kasbaoui, L., Harb, J., Bernard, S. \& Meflah2, K. Differences in Glycosylation State of Fibronectin from Two Rat Colon Carcinoma Cell Lines in Relation to Tumoral Progressiveness1. Cancer Res. 49, (1989).

47. Hakomori, S. \& Kannagi, R. Glycosphingolipids as tumor-associated and differentiation markers. J. Natl. Cancer Inst. 71, 231-51 (1983).

48. Varki, A., Kannagi, R., Toole, B. \& Stanley, P. Glycosylation Changes in Cancer. Essentials of Glycobiology (2015).

49. Ihara, S. et al. Prometastatic effect of $\mathrm{N}$-acetylglucosaminyltransferase $\mathrm{V}$ is due to modification and stabilization of active matriptase by adding beta 1-6 GIcNAc branching. J. Biol. Chem. 277, 16960-7 (2002).

50. Seidenfaden, R., Krauter, A., Schertzinger, F., Gerardy-Schahn, R. \& Hildebrandt, H. Polysialic acid directs tumor cell growth by controlling heterophilic neural cell adhesion molecule interactions. Mol.Cell Biol. 23, 5908-5918 (2003).

51. English, N. M., Lesley, J. F. \& Hyman, R. Site-specific de-N-glycosylation of CD44 can activate hyaluronan binding, and CD44 activation states show distinct threshold densities for hyaluronan binding. Cancer Res. 58, 3736-42 (1998).

52. Marsico, G., Russo, L., Quondamatteo, F. \& Pandit, A. Glycosylation and Integrin Regulation in Cancer. Trends in Cancer 4, 537-552 (2018).

53. Lavrsen, K. et al. Aberrantly glycosylated MUC1 is expressed on the surface of breast cancer cells and a target for antibody-dependent cell-mediated cytotoxicity. Glycoconj. J. 30, 22736 (2013).

54. Drake, R. R., Jones, E. E., Powers, T. W. \& Nyalwidhe, J. O. Altered Glycosylation in Prostate Cancer. in Advances in cancer research 126, 345-382 (2015).

55. Llop, E. et al. Improvement of Prostate Cancer Diagnosis by Detecting PSA GlycosylationSpecific Changes. Theranostics 6, 1190-204 (2016).

56. Tanaka, T. et al. Aberrant N-Glycosylation Profile of Serum Immunoglobulins is a Diagnostic Biomarker of Urothelial Carcinomas. Int. J. Mol. Sci. 18, 2632 (2017). 
57. Peracaula, R. et al. Altered glycosylation pattern allows the distinction between prostatespecific antigen (PSA) from normal and tumor origins. Glycobiology 13, 457-70 (2003).

58. Tabarés, G. et al. Different glycan structures in prostate-specific antigen from prostate cancer sera in relation to seminal plasma PSA. Glycobiology 16, 132-45 (2006).

59. Jia, G. et al. Alterations in expressed prostate secretion-urine PSA N-glycosylation discriminate prostate cancer from benign prostate hyperplasia. Oncotarget 8, 76987-76999 (2017).

60. Dal Moro, F., Valotto, C., Guttilla, A. \& Zattoni, F. Urinary Markers in the Everyday Diagnosis of Bladder Cancer. Urol. J. 80, 265-275 (2013).

61. Hautmann, S. H. et al. [Hyaluronic acid and hyaluronidase. 2 new bladder carcinoma markers]. Urologe. A 40, 121-6 (2001).

62. Cerliani, J. P., Blidner, A. G., Toscano, M. A., Croci, D. O. \& Rabinovich, G. A. Translating the 'Sugar Code' into Immune and Vascular Signaling Programs. Trends Biochem. Sci. 42, 255273 (2017).

63. Hirabayashi, J. \& Kasai, K. The family of metazoan metal-independent beta-galactosidebinding lectins: structure, function and molecular evolution. Glycobiology 3, 297-304 (1993).

64. Di Lella, S. et al. When galectins recognize glycans: from biochemistry to physiology and back again. Biochemistry 50, 7842-57 (2011).

65. Hirabayashi, J. et al. Oligosaccharide specificity of galectins: a search by frontal affinity chromatography. Biochim.Biophys.Acta 1572, 232-254 (2002).

66. Stowell, S. R. et al. Galectin-1, -2 , and -3 exhibit differential recognition of sialylated glycans and blood group antigens. J.Biol.Chem. 283, 10109-10123 (2008).

67. Greenspan, N. S. Dimensions of antigen recognition and levels of immunological specificity. Adv. Cancer Res. 80, 147-87 (2001).

68. Rabinovich, G. A., Toscano, M. A., Jackson, S. S. \& Vasta, G. R. Functions of cell surface galectin-glycoprotein lattices. Curr.Opin.Struct.Biol. 17, 513-520 (2007).

69. Stowell, S. R. et al. Ligand reduces galectin-1 sensitivity to oxidative inactivation by enhancing dimer formation. J. Biol. Chem. 284, 4989-99 (2009).

70. Leppanen, A., Stowell, S., Blixt, O. \& Cummings, R. D. Dimeric galectin-1 binds with high affinity to alpha2,3-sialylated and non-sialylated terminal $\mathrm{N}$-acetyllactosamine units on surface-bound extended glycans. J.Biol.Chem. 280, 5549-5562 (2005). 
71. Paz, A., Haklai, R., Elad-Sfadia, G., Ballan, E. \& Kloog, Y. Galectin-1 binds oncogenic H-Ras to mediate Ras membrane anchorage and cell transformation. Oncogene 20, 7486-7493 (2001).

72. Elad-Sfadia, G., Haklai, R., Balan, E. \& Kloog, Y. Galectin-3 augments K-Ras activation and triggers a Ras signal that attenuates ERK but not phosphoinositide 3-kinase activity. J. Biol. Chem. 279, 34922-30 (2004).

73. Salomonsson, E. et al. Monovalent interactions of galectin-1. Biochemistry 49, 9518-9532 (2010).

74. Camby, I., Le Mercier, M., Lefranc, F. \& Kiss, R. Galectin-1: a small protein with major functions. Glycobiology 16, 137R-157R (2006).

75. Voss, P. G. et al. Dissociation of the carbohydrate-binding and splicing activities of galectin1. Arch. Biochem. Biophys. 478, 18-25 (2008).

76. Hughes, R. C. Secretion of the galectin family of mammalian carbohydrate-binding proteins. Biochim.Biophys.Acta 1473, 172-185 (1999).

77. Leffler, H., Carlsson, S., Hedlund, M., Qian, Y. \& Poirier, F. Introduction to galectins. Glycoconj.J. 19, 433-440 (2004).

78. Compagno, D. et al. Galectins: major signaling modulators inside and outside the cell. Curr. Mol. Med. 14, 630-51 (2014).

79. Balan, V., Nangia-Makker, P. \& Raz, A. Galectins as cancer biomarkers. Cancers (Basel). 2, 592-610 (2010).

80. Jones, J. L. et al. Galectin-3 is associated with prostasomes in human semen. Glycoconj. J. 27, 227-236 (2010).

81. Thijssen, V. L., Heusschen, R., Caers, J. \& Griffioen, A. W. Galectin expression in cancer diagnosis and prognosis: A systematic review. Biochim. Biophys. Acta 1855, 235-47 (2015).

82. Thijssen, V. L. et al. Tumor cells secrete galectin-1 to enhance endothelial cell activity. Cancer Res. 70, 6216-24 (2010).

83. Manzi, M. et al. Galectin-1 Controls the Proliferation and Migration of Liver Sinusoidal Endothelial Cells and Their Interaction With Hepatocarcinoma Cells. J. Cell. Physiol. 231, 1522-1533 (2016).

84. Yamaoka, K. et al. Expression of galectin-1 mRNA correlates with the malignant potential of human gliomas and expression of antisense galectin-1 inhibits the growth of 9 glioma cells. J.Neurosci.Res. 59, 722-730 (2000). 
85. Orozco, C. A. et al. Targeting galectin-1 inhibits pancreatic cancer progression by modulating tumor-stroma crosstalk. Proc. Natl. Acad. Sci. 115, E3769-E3778 (2018).

86. Rabinovich, G. A. et al. Specific inhibition of T-cell adhesion to extracellular matrix and proinflammatory cytokine secretion by human recombinant galectin-1. Immunology 97 , 100-106 (1999).

87. Rabinovich, G. A., Toscano, M. A., Ilarregui, J. M. \& Rubinstein, N. Shedding light on the immunomodulatory properties of galectins: novel regulators of innate and adaptive immune responses. Glycoconj.J. 19, 565-573 (2004).

88. Liu, F. T. \& Rabinovich, G. A. Galectins as modulators of tumour progression. Nat.Rev.Cancer 5, 29-41 (2005).

89. Gao, X., Liu, J., Liu, X., Li, L. \& Zheng, J. Cleavage and phosphorylation: important posttranslational modifications of galectin-3. Cancer Metastasis Rev. 36, 367-374 (2017).

90. Mazurek, N., Conklin, J., Byrd, J. C., Raz, A. \& Bresalier, R. S. Phosphorylation of the betagalactoside-binding protein galectin-3 modulates binding to its ligands. J. Biol. Chem. 275, $36311-5$ (2000).

91. Yoshii, T. et al. Galectin-3 phosphorylation is required for its anti-apoptotic function and cell cycle arrest. J. Biol. Chem. 277, 6852-7 (2002).

92. Danguy, A., Camby, I. \& Kiss, R. Galectins and cancer. Biochim.Biophys.Acta 1572, 285-293 (2002).

93. van den, B. F., Califice, S. \& Castronovo, V. Expression of galectins in cancer: a critical review. Glycoconj.J. 19, 537-542 (2004).

94. Yang, R. Y., Rabinovich, G. A. \& Liu, F. T. Galectins: structure, function and therapeutic potential. Expert.Rev.Mol.Med. 10, e17 (2008).

95. Teichberg, V. I., Silman, I., Beitsch, D. D. \& Resheff, G. A beta-D-galactoside binding protein from electric organ tissue of Electrophorus electricus. Proc. Natl. Acad. Sci. U. S. A. 72, 1383-7 (1975).

96. Raz, A. \& Lotan, R. Endogenous galactoside-binding lectins: a new class of functional tumor cell surface molecules related to metastasis. Cancer Metastasis Rev. 6, 433-52 (1987).

97. Raz, A. \& Lotan, R. Lectin-like activities associated with human and murine neoplastic cells. Cancer Res. 41, 3642-7 (1981).

98. Elad-Sfadia, G., Haklai, R., Ballan, E., Gabius, H. J. \& Kloog, Y. Galectin-1 augments Ras activation and diverts Ras signals to Raf-1 at the expense of phosphoinositide 3-kinase. 
J.Biol.Chem. 277, 37169-37175 (2002).

99. Andre, S. et al. Galectins-1 and -3 and their ligands in tumor biology. Non-uniform properties in cell-surface presentation and modulation of adhesion to matrix glycoproteins for various tumor cell lines, in biodistribution of free and liposome-bound galectins and in thei. J.Cancer Res.Clin.Oncol. 125, 461-474 (1999).

100. Gu, M., Wang, W., Song, W. K., Cooper, D. N. \& Kaufman, S. J. Selective modulation of the interaction of alpha 7 beta 1 integrin with fibronectin and laminin by L-14 lectin during skeletal muscle differentiation. J.Cell Sci. 107 ( Pt 1, 175-181 (1994).

101. Shimura, T. et al. Implication of Galectin-3 in Wnt Signaling. Cancer Res. 65, 3535-3537 (2005).

102. Shimura, T. et al. Galectin-3, a Novel Binding Partner of $\beta$-Catenin. Cancer Res. 64, 63636367 (2004).

103. Martinez-Bosch, N. et al. Galectin-1 Drives Pancreatic Carcinogenesis through Stroma Remodeling and Hedgehog Signaling Activation. Cancer Res. 74, 3512-3524 (2014).

104. Rabinovich, G. A. et al. Induction of allogenic T-cell hyporesponsiveness by galectin-1mediated apoptotic and non-apoptotic mechanisms. Cell Death.Differ. 9, 661-670 (2002).

105. He, J. \& Baum, L. G. Presentation of galectin-1 by extracellular matrix triggers T cell death. J.Biol.Chem. 279, 4705-4712 (2004).

106. Ebrahim, A. H. et al. Galectins in cancer: carcinogenesis, diagnosis and therapy. Ann. Transl. Med. 2, 88 (2014).

107. Rabinovich, G. A. \& Conejo-García, J. R. Shaping the Immune Landscape in Cancer by Galectin-Driven Regulatory Pathways. J. Mol. Biol. 428, 3266-3281 (2016).

108. Colnot, C., Fowlis, D., Ripoche, M. A., Bouchaert, I. \& Poirier, F. Embryonic implantation in galectin 1/galectin 3 double mutant mice. Dev.Dyn. 211, 306-313 (1998).

109. Wang, W., Park, J. W., Wang, J. L. \& Patterson, R. J. Immunoprecipitation of spliceosomal RNAs by antisera to galectin-1 and galectin-3. Nucleic Acids Res. 34, 5166-74 (2006).

110. Demydenko, D. \& Berest, I. Expression of galectin-1 in malignant tumors. Exp.Oncol. 31, 7479 (2009).

111. Blanchard, H., Bum-Erdene, K., Bohari, M. H. \& Yu, X. Galectin-1 inhibitors and their potential therapeutic applications: a patent review. Expert Opin. Ther. Pat. 26, 537-554 (2016).

112. Laderach, D. J. et al. A Unique Galectin Signature in Human Prostate Cancer Progression 
Suggests Galectin-1 as a Key Target for Treatment of Advanced Disease. Cancer Res. 73, 8696 (2013).

113. Compagno, D. et al. Glycans and galectins in prostate cancer biology, angiogenesis and metastasis. Glycobiology 24, 899-906 (2014).

114. Ellerhorst, J., Troncoso, P., Xu, X. C., Lee, J. \& Lotan, R. Galectin-1 and galectin-3 expression in human prostate tissue and prostate cancer. Urol. Res. 27, 362-7 (1999).

115. Ellerhorst, J., Nguyen, T., Cooper, D. N., Lotan, D. \& Lotan, R. Differential expression of endogenous galectin-1 and galectin-3 in human prostate cancer cell lines and effects of overexpressing galectin-1 on cell phenotype. Int.J.Oncol. 14, 217-224 (1999).

116. Clausse, N., van den Brûle, F., Waltregny, D., Garnier, F. \& Castronovo, V. Galectin-1 expression in prostate tumor-associated capillary endothelial cells is increased by prostate carcinoma cells and modulates heterotypic cell-cell adhesion. Angiogenesis 3, 317-25 (1999).

117. van den Brûle, F. A., Waltregny, D. \& Castronovo, V. Increased expression of galectin-1 in carcinoma-associated stroma predicts poor outcome in prostate carcinoma patients. J. Pathol. 193, 80-7 (2001).

118. He, J. \& Baum, L. G. Endothelial cell expression of galectin-1 induced by prostate cancer cells inhibits T-cell transendothelial migration. Lab. Investig. 86, 578-590 (2006).

119. Valenzuela, H. F. et al. O-Glycosylation Regulates LNCaP Prostate Cancer Cell Susceptibility to Apoptosis Induced by Galectin-1. Cancer Res. 67, 6155-6162 (2007).

120. Petrosyan, A., Holzapfel, M. S., Muirhead, D. E. \& Cheng, P.-W. Restoration of Compact Golgi Morphology in Advanced Prostate Cancer Enhances Susceptibility to Galectin-1Induced Apoptosis by Modifying Mucin O-Glycan Synthesis. Mol. Cancer Res. 12, 17041716 (2014).

121. Shih, T.-C. et al. Targeting Galectin-1 impairs castration-resistant prostate cancer progression and invasion. Clin. Cancer Res. clincanres.0157.2018 (2018). doi:10.1158/10780432.CCR-18-0157

122. Andersen, H., Jensen, O. N., Moiseeva, E. P. \& Eriksen, E. F. A proteome study of secreted prostatic factors affecting osteoblastic activity: galectin-1 is involved in differentiation of human bone marrow stromal cells. J.Bone Miner.Res. 18, 195-203 (2003).

123. Jaworski, F. M. et al. In VivoHemin Conditioning Targets the Vascular and Immunologic Compartments and Restrains Prostate Tumor Development. Clin. Cancer Res. 23, 5135- 
5148 (2017).

124. Corapi, E., Carrizo, G., Compagno, D. \& Laderach, D. Endogenous Galectin-1 in T Lymphocytes Regulates Anti-prostate Cancer Immunity. Front. Immunol. 9, 2190 (2018).

125. Pacis, R. A. et al. Decreased galectin-3 expression in prostate cancer. Prostate 44, 118-23 (2000).

126. Merseburger, A. S. et al. Involvement of decreased Galectin-3 expression in the pathogenesis and progression of prostate cancer. Prostate 68, 72-77 (2008).

127. Wang, Y. et al. Regulation of Prostate Cancer Progression by Galectin-3. Am. J. Pathol. 174, 1515-1523 (2009).

128. de Melo-Júnior, M. R., Araújo-Filho, J. L. S., Lins, C. A. B., de Pontes-Filho, N. T. \& de Carvalho, L. B. Immobilization of anti-galectin-3 onto polysiloxane-polyvinyl alcohol disks for tumor prostatic diseases diagnosis. Appl. Biochem. Biotechnol. 160, 2198-207 (2010).

129. Knapp, J. S. et al. Galectin-3 expression in prostate cancer and benign prostate tissues: correlation with biochemical recurrence. World J. Urol. 31, 351-8 (2013).

130. Geisler, C. et al. Identification and Validation of Potential New Biomarkers for Prostate Cancer Diagnosis and Prognosis Using 2D-DIGE and MS. Biomed Res. Int. 2015, 1-23 (2015).

131. van den Brûle, F. A., Waltregny, D., Liu, F. T. \& Castronovo, V. Alteration of the cytoplasmic/nuclear expression pattern of galectin-3 correlates with prostate carcinoma progression. Int. J. cancer 89, 361-7 (2000).

132. Ahmed, H., Banerjee, P. P. \& Vasta, G. R. Differential expression of galectins in normal, benign and malignant prostate epithelial cells: silencing of galectin-3 expression in prostate cancer by its promoter methylation. Biochem. Biophys. Res. Commun. 358, 241-6 (2007).

133. Califice, S., Castronovo, V., Bracke, M. \& van den, B. F. Dual activities of galectin-3 in human prostate cancer: tumor suppression of nuclear galectin-3 vs tumor promotion of cytoplasmic galectin-3. Oncogene 23, 7527-7536 (2004).

134. Fukumori, T. et al. Galectin-3 regulates mitochondrial stability and antiapoptotic function in response to anticancer drug in prostate cancer. Cancer Res. 66, 3114-9 (2006).

135. Wang, Y., Nangia-Makker, P., Balan, V., Hogan, V. \& Raz, A. Calpain activation through galectin-3 inhibition sensitizes prostate cancer cells to cisplatin treatment. Cell Death Dis. 1, e101 (2010).

136. Dondoo, T.-O. et al. Galectin-3 Is Implicated in Tumor Progression and Resistance to Antiandrogen Drug Through Regulation of Androgen Receptor Signaling in Prostate Cancer. 
Anticancer Res. 37, 125-134 (2017).

137. Guha, P. et al. Cod glycopeptide with picomolar affinity to galectin-3 suppresses T-cell apoptosis and prostate cancer metastasis. Proc. Natl. Acad. Sci. U. S. A. 110, 5052-7 (2013).

138. Glinsky, V. V et al. The role of Thomsen-Friedenreich antigen in adhesion of human breast and prostate cancer cells to the endothelium. Cancer Res. 61, 4851-7 (2001).

139. Glinsky, V. V et al. Intravascular metastatic cancer cell homotypic aggregation at the sites of primary attachment to the endothelium. Cancer Res. 63, 3805-11 (2003).

140. Meng, F., Joshi, B. \& Nabi, I. R. Galectin-3 Overrides PTRF/Cavin-1 Reduction of PC3 Prostate Cancer Cell Migration. PLoS One 10, e0126056 (2015).

141. Farhad, M., Rolig, A. S. \& Redmond, W. L. The role of Galectin-3 in modulating tumor growth and immunosuppression within the tumor microenvironment. Oncoimmunology 7, e1434467 (2018).

142. Lee, Y.-C. et al. Secretome analysis of an osteogenic prostate tumor identifies complex signaling networks mediating cross-talk of cancer and stromal cells within the tumor microenvironment. Mol. Cell. Proteomics 14, 471-83 (2015).

143. Nakajima, K. et al. Galectin-3 Cleavage Alters Bone Remodeling: Different Outcomes in Breast and Prostate Cancer Skeletal Metastasis. Cancer Res. 76, 1391-1402 (2016).

144. Pienta, K. J. et al. Inhibition of spontaneous metastasis in a rat prostate cancer model by oral administration of modified citrus pectin. J.Natl.Cancer Inst. 87, 348-353 (1995).

145. Glinskii, O. V et al. Inhibition of prostate cancer bone metastasis by synthetic TF antigen mimic/galectin-3 inhibitor lactulose-L-leucine. Neoplasia 14, 65-73 (2012).

146. Nakajima, K. et al. Positive associations between galectin-3 and PSA levels in prostate cancer patients: a prospective clinical study-I. Oncotarget 7, 82266-82272 (2016).

147. Nakajima, K. et al. The influence of PSA autoantibodies in prostate cancer patients: a prospective clinical study-II. Oncotarget 8, 17643-17650 (2017).

148. Ochieng, J. et al. Galectin-3 is a novel substrate for human matrix metalloproteinases-2 and -9. Biochemistry 33, 14109-14 (1994).

149. Saraswati, S. et al. Galectin-3 is a substrate for prostate specific antigen (PSA) in human seminal plasma. Prostate 71, 197-208 (2011).

150. Yang, Y. et al. Treatment of prostate carcinoma with (galectin-3)-targeted HPMA copolymer-(G3-C12)-5-Fluorouracil conjugates. Biomaterials 33, 2260-71 (2012).

151. Glinsky, V. V \& Raz, A. Modified citrus pectin anti-metastatic properties: one bullet, 
multiple targets. Carbohydr. Res. 344, 1788-91 (2009).

152. Conti, S. et al. Modified Citrus Pectin as a Potential Sensitizer for Radiotherapy in Prostate Cancer. Integr. Cancer Ther. 17, 1225-34 (2018).

153. Tsai, C.-H. et al. Metastatic Progression of Prostate Cancer Is Mediated by Autonomous Binding of Galectin-4- O -Glycan to Cancer Cells. Cancer Res. 76, 5756-5767 (2016).

154. Tzeng, S.-F. et al. O-Glycosylation-mediated signaling circuit drives metastatic castrationresistant prostate cancer. FASEB J. fj201800687 (2018).

155. Labrie, M. et al. A Mutation in the Carbohydrate Recognition Domain Drives a Phenotypic Switch in the Role of Galectin-7 in Prostate Cancer. PLoS One 10, e0131307 (2015).

156. Su, Z. Z. et al. Surface-epitope masking and expression cloning identifies the human prostate carcinoma tumor antigen gene PCTA-1 a member of the galectin gene family. Proc. Natl. Acad. Sci. U. S. A. 93, 7252-7 (1996).

157. Gopalkrishnan, R. V et al. Molecular characterization of prostate carcinoma tumor antigen1, PCTA-1, a human galectin-8 related gene. Oncogene 19, 4405-16 (2000).

158. Danguy, A. et al. Immunohistochemical profile of galectin-8 expression in benign and malignant tumors of epithelial, mesenchymatous and adipous origins, and of the nervous system. Histol. Histopathol. 16, 861-8 (2001).

159. Nguyen, M. C. et al. Antibody responses to galectin-8, TARP and TRAP1 in prostate cancer patients treated with a GM-CSF-secreting cellular immunotherapy. Cancer Immunol. Immunother. 59, 1313-23 (2010).

160. Gentilini, L. D. et al. Stable and high expression of Galectin-8 tightly controls metastatic progression of prostate cancer. Oncotarget 8, 44654-44668 (2017).

161. GuhaThakurta, D. et al. Humoral Immune Response against Nontargeted Tumor Antigens after Treatment with Sipuleucel-T and Its Association with Improved Clinical Outcome. Clin. Cancer Res. 21, 3619-30 (2015).

162. Cindolo, L. et al. galectin-1 and galectin-3 expression in human bladder transitional-cell carcinomas. Int.J.Cancer 84, 39-43 (1999).

163. Chuang, C.-H. et al. Lab on a chip for multiplexed immunoassays to detect bladder cancer using multifunctional dielectrophoretic manipulations. Lab Chip 15, 3056-3064 (2015).

164. Shen, K.-H. et al. Role of galectin-1 in urinary bladder urothelial carcinoma cell invasion through the JNK pathway. Cancer Sci. 107, 1390-1398 (2016).

165. Li, C.-F. et al. Proteomic Identification of the Galectin-1-Involved Molecular Pathways in 
Urinary Bladder Urothelial Carcinoma. Int. J. Mol. Sci. 19, 1242 (2018).

166. Fang, T. et al. Modified citrus pectin inhibited bladder tumor growth through downregulation of galectin-3. Acta Pharmacol. Sin. 39, 1885-1893 (2018).

167. Matsui, Y. et al. Sensitizing Effect of Galectin-7 in Urothelial Cancer to Cisplatin through the Accumulation of Intracellular Reactive Oxygen Species. Cancer Res. 67, 1212-1220 (2007).

168. Kramer, M. W. et al. Decreased galectin-8 is a strong marker for recurrence in urothelial carcinoma of the bladder. Urol. Int. 87, 143-50 (2011).

169. Griffioen, A. W. \& Thijssen, V. L. Galectins in tumor angiogenesis. Ann. Transl. Med. 2, 90 (2014).

170. Méndez-Huergo, S. P., Blidner, A. G. \& Rabinovich, G. A. Galectins: emerging regulatory checkpoints linking tumor immunity and angiogenesis. Curr. Opin. Immunol. 45, 8-15 (2017).

171. Tsuboi, S. et al. A novel strategy for evasion of NK cell immunity by tumours expressing core2 O-glycans. EMBO J. 30, 3173-85 (2011).

172. Wang, W. et al. Tumor-released Galectin-3, a soluble inhibitory ligand of human NKp30, plays an important role in tumor escape from NK cell attack. J. Biol. Chem. 289, 33311-9 (2014).

173. Li, H. et al. Tim-3/galectin-9 signaling pathway mediates T-cell dysfunction and predicts poor prognosis in patients with hepatitis B virus-associated hepatocellular carcinoma. Hepatology 56, 1342-51 (2012).

174. Kang, C.-W. et al. Apoptosis of tumor infiltrating effector TIM-3+CD8+ T cells in colon cancer. Sci. Rep. 5, 15659 (2015).

175. Dardalhon, V. et al. Tim-3/galectin-9 pathway: regulation of Th1 immunity through promotion of CD11b+Ly-6G+ myeloid cells. J. Immunol. 185, 1383-92 (2010).

176. US National Library of Medicine. ClinicalTrials.gov https://clinicaltrials.gov/ct2/show/NCT01724320 (2012)

177. Jean-Pierre Delord, Ahmad Awada, Eric Raymond, François Lokiec, et al. A first-in-man Phase I study of the galectin-1 (gal-1) inhibitor OTX008 given subcutaneously as a single agent to patients with advanced solid tumors. Mol Cancer Ther 12, Abstract A72 (2013).

178. US National Library of Medicine. ClinicalTrials.gov https://clinicaltrials.gov/ct2/show/NCT054977 (2003).

179. US National Library of Medicine. ClinicalTrials.gov 
https://clinicaltrials.gov/ct2/show/NCT00110721 (2005).

180. Klyosov, A., Zomer, E. \& Platt, D. DAVANAT ${ }^{\circledR}$ (GM-CT-01) and Colon Cancer: Preclinical and Clinical (Phase I and II) Studies. in Glycobiology and Drug Design (ed. ACS Symposium Series) 89-130 (American Chemical Society, 2012).

181. US National Library of Medicine. ClinicalTrials.gov https://clinicaltrials.gov/ct2/show/NCT01723813 (2012).

182. Linch, S. et al. Galectin-3 inhibition using novel inhibitor GR-MD-02 improves survival and immune function while reducing tumor vasculature. J. Immunother. Cancer 3, P306 (2015).

183. US National Library of Medicine. ClinicalTrials.gov https://clinicaltrials.gov/ct2/show/NCT02117362 (2015).

184. US National Library of Medicine. ClinicalTrials.gov https://clinicaltrials.gov/ct2/show/NCT02575404 (2015).

185. Pérez, C. V et al. Dual roles of endogenous and exogenous galectin-1 in the control of testicular immunopathology. Sci. Rep. 5, 12259 (2015).

186. Paclik, D. et al. Galectin-2 induces apoptosis of lamina propria T lymphocytes and ameliorates acute and chronic experimental colitis in mice. J. Mol. Med. (Berl). 86, 1395406 (2008).

187. López, E. et al. Inhibition of chronic airway inflammation and remodeling by galectin-3 gene therapy in a murine model. J. Immunol. 176, 1943-50 (2006).

188. Watanabe, M. et al. Clinical significance of circulating galectins as colorectal cancer markers. Oncol. Rep. 25, 1217-26 (2011).

189. Verschuere, T. et al. Altered galectin-1 serum levels in patients diagnosed with high-grade glioma. J. Neurooncol. 115, 9-17 (2013).

190. Ouyang, J. et al. Galectin-1 serum levels reflect tumor burden and adverse clinical features in classical Hodgkin lymphoma. Blood 121, 3431-3433 (2013).

191. Aggarwal, S., Sharma, S. C. \& Das, S. N. Galectin-1 and galectin-3: Plausible tumour markers for oral squamous cell carcinoma and suitable targets for screening high-risk population. Clin. Chim. Acta 442, 13-21 (2015).

192. Kaneko, N. et al. Potential tumor markers of renal cell carcinoma: $\alpha$-Enolase for postoperative follow up, and galectin-1 and galectin-3 for primary detection. Int. J. Urol. 20, 530-535 (2013).

193. Waalkes, S., Merseburger, A. S., Simon, A., Serth, J. \& Kuczyk, M. A. Galectin-Expression an 
urologischen Tumoren. Urologe 49, 387-391 (2010).

194. El Gendy, H. et al. Galectin 3 for the diagnosis of bladder cancer. Arab J. Urol. 12, 178-81 (2014).

195. Poirier, F. \& Robertson, E. J. Normal development of mice carrying a null mutation in the gene encoding the L14 S-type lectin. Development 119, 1229-1236 (1993).

196. Sundblad, V., Morosi, L. G., Geffner, J. R. \& Rabinovich, G. A. Galectin-1: A Jack-of-All-Trades in the Resolution of Acute and Chronic Inflammation. J. Immunol. 199, 3721-3730 (2017).

197. Arthur, C. M., Baruffi, M. D., Cummings, R. D. \& Stowell, S. R. Evolving mechanistic insights into galectin functions. Methods Mol. Biol. 1207, 1-35 (2015).

198. Croci, D. O. et al. Disrupting galectin-1 interactions with N-glycans suppresses hypoxiadriven angiogenesis and tumorigenesis in Kaposi's sarcoma. J. Exp. Med. 209, 1985-2000 (2012).

199. Ouyang, J. et al. Viral induction and targeted inhibition of galectin-1 in EBV+ posttransplant lymphoproliferative disorders. Blood 117, 4315-22 (2011).

200. Becher, O. J. \& Holland, E. C. Genetically engineered models have advantages over xenografts for preclinical studies. Cancer Res. 66, 3355-8, NaN-9 (2006).

201. Day, C.-P., Merlino, G. \& Van Dyke, T. Preclinical mouse cancer models: a maze of opportunities and challenges. Cell 163, 39-53 (2015).

202. Grabowska, M. M. et al. Mouse models of prostate cancer: picking the best model for the question. Cancer Metastasis Rev. 33, 377-97 (2014).

203. Rea, D. et al. Mouse Models in Prostate Cancer Translational Research: From Xenograft to PDX. Biomed Res. Int. 2016, 9750795 (2016).

204. Kobayashi, T., Owczarek, T. B., McKiernan, J. M. \& Abate-Shen, C. Modelling bladder cancer in mice: opportunities and challenges. Nat. Rev. Cancer 15, 42-54 (2015).

205. Eisenstein, M. Organoids: the body builders. Nat. Methods 15, 19-22 (2018).

206. Vela, I. \& Chen, Y. Prostate cancer organoids: a potential new tool for testing drug sensitivity. Expert Rev. Anticancer Ther. 15, 261-3 (2015).

207. Gao, D. et al. Organoid Cultures Derived from Patients with Advanced Prostate Cancer. Cell 159, 176-187 (2014).

208. Karthaus, W. R. et al. Identification of multipotent luminal progenitor cells in human prostate organoid cultures. Cell 159, 163-175 (2014).

209. Lee, S. H. et al. Tumor Evolution and Drug Response in Patient-Derived Organoid Models of 
Bladder Cancer. Cell 173, 515-528.e17 (2018).

210. Yoshida, T., Singh, A. K., Bishai, W. R., McConkey, D. J. \& Bivalacqua, T. J. Organoid culture of bladder cancer cells. Investig. Clin. Urol. 59, 149 (2018).

211. Wang, S., Gao, D. \& Chen, Y. The potential of organoids in urological cancer research. Nat. Rev. Urol. 14, 401-414 (2017).

212. Kantoff, P. W. et al. Sipuleucel-T immunotherapy for castration-resistant prostate cancer. N. Engl. J. Med. 363, 411-22 (2010).

213. Goswami, S., Aparicio, A. \& Subudhi, S. K. Immune Checkpoint Therapies in Prostate Cancer. Cancer J. 22, 117-20 (2016).

214. Beer, T. M. et al. Randomized, Double-Blind, Phase III Trial of I pilimumab Versus Placebo in Asymptomatic or Minimally Symptomatic Patients With Metastatic Chemotherapy-Naive Castration-Resistant Prostate Cancer. J. Clin. Oncol. 35, 40-47 (2017).

215. Fakhrejahani, F. et al. Avelumab in metastatic castration-resistant prostate cancer (mCRPC). J. Clin. Oncol. 35, 159-159 (2017). 


\section{FIGURES}

Figure 1

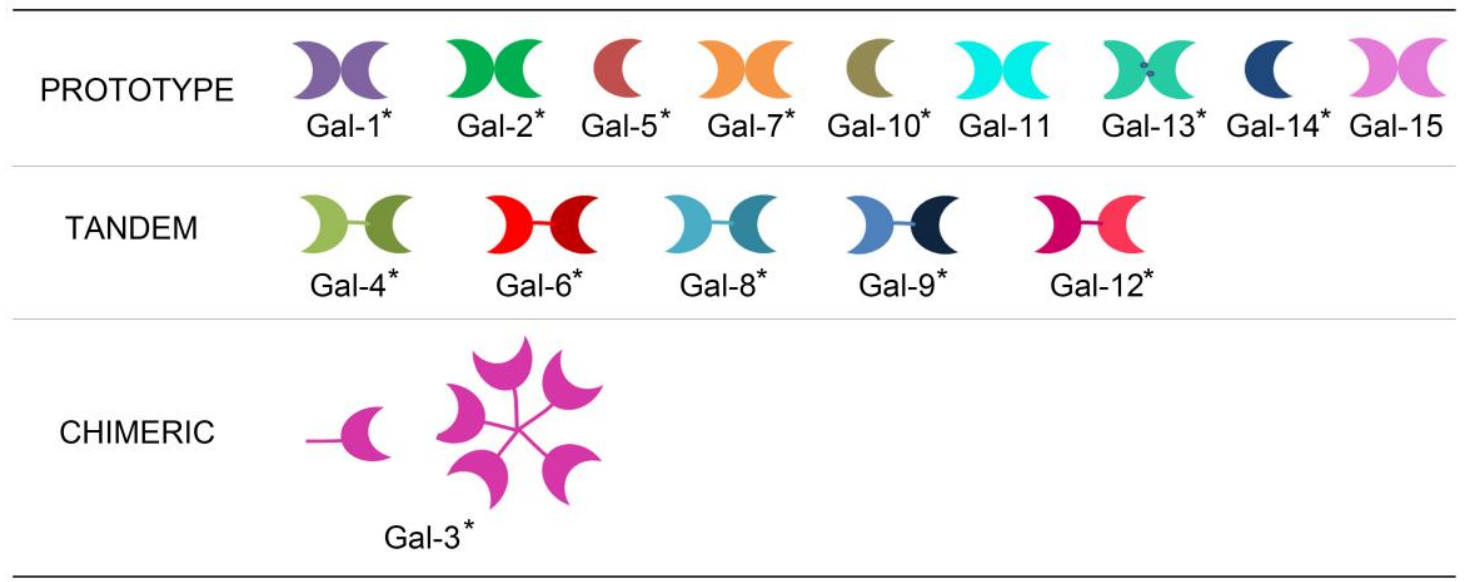

Figure 2

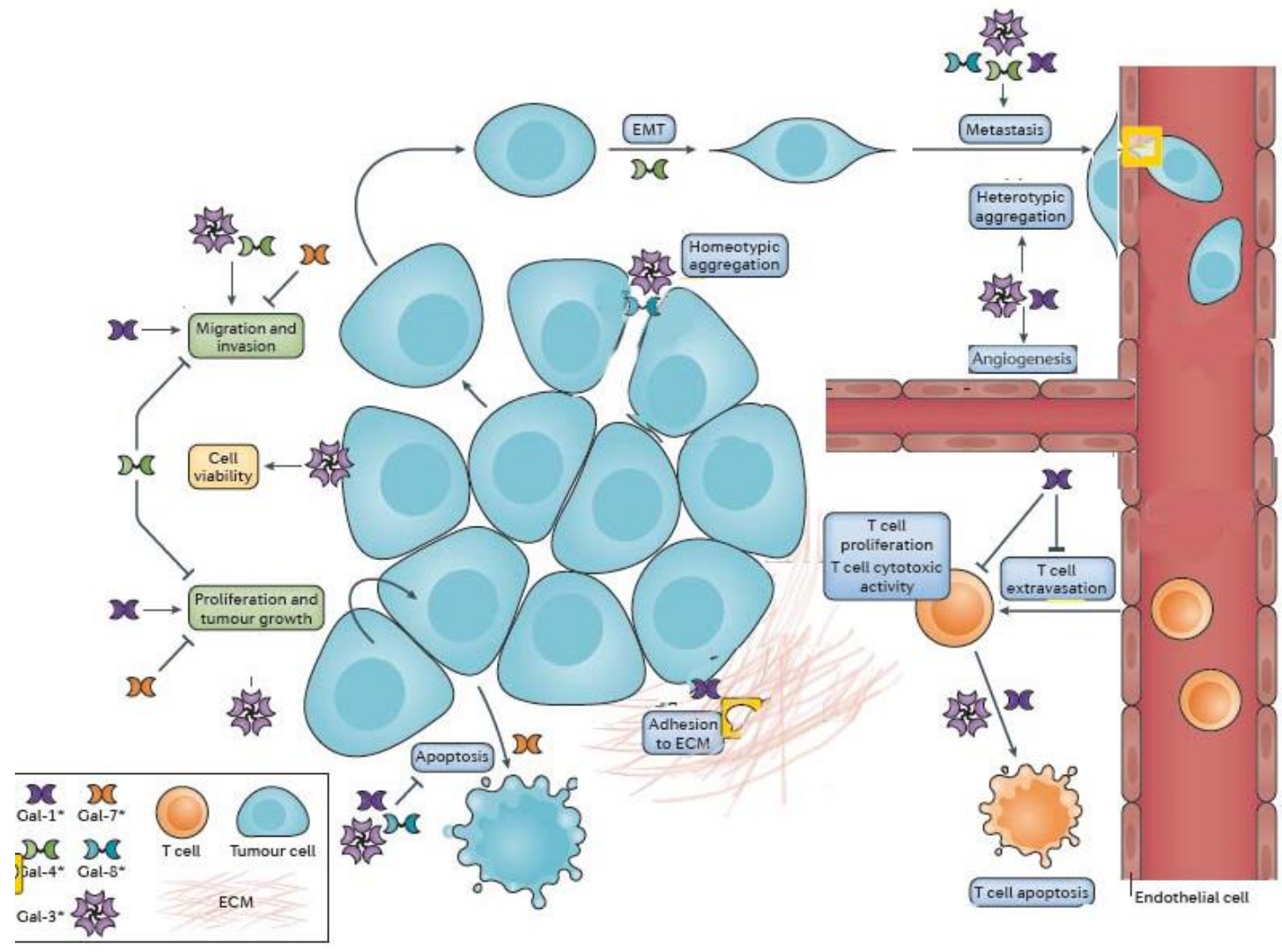

